\title{
Double and triple-harmonic RF buckets and their use for bunch squeezing in AGS
}

\section{C.J. Gardner}

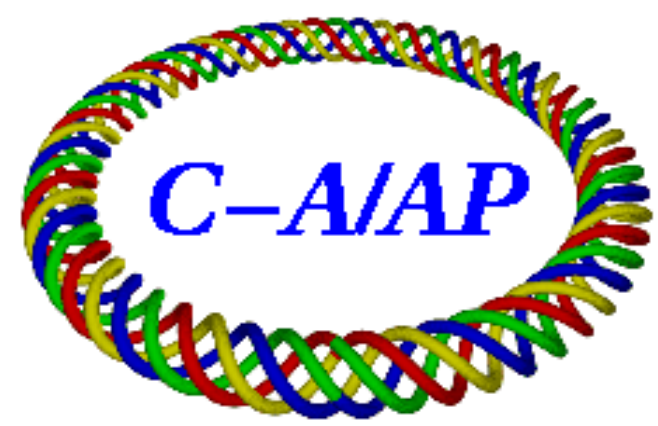

Collider-Accelerator Department Brookhaven National Laboratory

Upton, NY 11973

\section{U.S. Department of Energy \\ Office of Science, Office of Nuclear Physics}

Notice: This document has been authorized by employees of Brookhaven Science Associates, LLC under Contract No. DE-SC0012704 with the U.S. Department of Energy. The United States Government retains a nonexclusive, paid-up, irrevocable, world-wide license to publish or reproduce the published form of this document, or allow others to do so, for United States Government purposes. 


\title{
Double and Triple-Harmonic RF buckets and their use for Bunch Squeezing in AGS
}

\author{
C.J. Gardner
}

August 24, 2016

For the past several years we have merged bunches in AGS in order to achieve the desired intensity per bunch prior to injection into RHIC $[1,2]$. The merging is done on a flat porch at or above AGS injection energy. Because the merges involve the reduction of the RF harmonic number by a factor of 2 (for a 2 to 1 merge) and then a factor of 3 (for a 3 to 1 merge), one requires $\mathrm{RF}$ frequencies $6 h f_{s}, 3 h f_{s}, 2 h f_{s}$ and $h f_{s}$, where $f_{s}$ is the revolution frequency on the porch and $h=4$ is the fundamental harmonic number. The standard AGS RF cavities cannot operate at the lowest frequencies $2 h f_{s}$ and $h f_{s}$; these are provided by two modified cavities. Upon completion of the merges, the bunches are sitting in harmonic $h$ buckets. In order to be accelerated they need to be squeezed into harmonic $3 h$ buckets. This is accomplished by producing a double-harmonic bucket in which harmonics $h$ and $2 h$ act in concert, and then a triple-harmonic bucket in which harmonics $h, 2 h$, and $3 h$ act in concert. Simulations have shown [2] that the squeeze presents an acceptance bottleneck which limits the longitudinal emittance that can be put into the harmonic $3 h$ bucket.

In this note the areas of the double and triple-harmonic buckets are calculated explicitly and it is shown that these go through a minimum as the $\mathrm{RF}$ voltages are raised to the desired values. Several RF voltage ranges are examined and the acceptance bottleneck is determined for each of these. The results are in good agreement with those obtained by simulation in [2].

Sections 1-5 serve as the setup for the area calculations that are carried out in Sections 6-10. The double-harmonic bucket area is calculated in Sections 6-8. Here it is shown that the minimum area is easily obtained from the unstable fixed point phase that satisfies the transcendental 
equation

$$
\xi+\tan \xi=0
$$

Calculations of the triple-harmonic area and its minimum are carried out in Sections 9 and 10. Here the required integration is done numerically.

In Sections 11 and 12 a short review of the most recent setup for merging and squeezing Au77+ bunches in AGS is given.

Finally, in Section 13 the acceptance bottleneck for Au77+ bunches in AGS is calculated for several RF voltage ranges. The main result is that the RF voltages for the low-frequency harmonic $h$ and $2 h$ cavities both must be at least $22 \mathrm{kV}$ in order to achieve an acceptance of $0.6 \mathrm{eV} \mathrm{s}$ per nucleon. If the harmonic $h$ and $2 h$ voltages are 15 and $22 \mathrm{kV}$, respectively, then the acceptance is reduced to $0.548 \mathrm{eVs}$ per nucleon.

(On a first reading, one may wish to skip over Sections 1-10 and start with Section 11; or one may choose to look just at the Figures, which start on page 29.)

\section{Synchronous Parameters}

Let $2 \pi R$ and $\rho$ be the circumference and radius-of-curvature of the design orbit in a given ring, and let $B$ and $2 \pi R_{s}$ be the magnetic field and orbit circumference for the synchronous particle. We assume that $B$ and $R_{s}$ are given and calculate the other synchronous particle parameters in terms of these. The radius-of-curvature of the synchronous particle is

$$
\rho_{s}=\rho\left(R_{s} / R\right)^{1 / \alpha}
$$

where

$$
\alpha=\frac{1}{\gamma_{t}^{2}}
$$

is the "momentum compaction" factor and $\gamma_{t}$ is the transition gamma of the ring. The momentum of the synchronous particle is given by

$$
c p_{s}=e Q B \rho_{s}
$$

where $e$ is the proton charge and $e Q$ is the charge of the particle. The energy is

$$
E_{s}=\sqrt{\left(c p_{s}\right)^{2}+m^{2} c^{4}}
$$


where $m$ is the mass of the particle. The synchronous $\beta, \gamma$ and angular velocity are

$$
\beta_{s}=c p_{s} / E_{s}, \quad \gamma_{s}=E_{s} /\left(m c^{2}\right), \quad \omega_{s}=c \beta_{s} / R_{s} .
$$

The revolution period and frequency of the synchronous particle are

$$
T_{s}=\frac{2 \pi}{\omega_{s}}, \quad f_{s}=\frac{1}{T_{s}}
$$

We use $T^{s}$ to denote the arrival time (at an RF cavity) of the synchronous particle. We also define the phase slip factor

$$
\eta_{s}=\frac{1}{\gamma_{t}^{2}}-\frac{1}{\gamma_{s}^{2}}
$$

We assume that the bunch squeezing takes place on a porch below transition. Thus

$$
\frac{d B}{d t}=0
$$

and

$$
\eta_{s}<0
$$

\section{Particle Motion}

The hamiltonian

$$
H(\phi, W)=\frac{1}{2} a W^{2}+U(\phi)
$$

generates motion that approximates that of the asynchronous particle.

Here

$$
\phi=h \omega_{s}\left(T-T^{s}\right), \quad W=\frac{E-E_{s}}{h \omega_{s}}
$$

and

$$
a=\left(\frac{h^{2} \omega_{s}^{2} \eta_{s}}{\beta_{s}^{2} E_{s}}\right)=\left(\frac{h^{2} c^{2} \eta_{s}}{R_{s}^{2} E_{s}}\right) .
$$

The positive integer $h$ is the fundamental RF harmonic number. The time and energy deviations of the asynchronous particle are $T-T^{s}$ and $E-E_{s}$, respectively. Since we are considering the motion on a porch below transition, we have

$$
a<0 \text {. }
$$


The "potential" $U(\phi)$ is given by

$$
\frac{\partial U}{\partial \phi}=-F(\phi)
$$

where

$$
F(\phi)=A_{1} \sin \phi+A_{2} \sin 2 \phi+A_{3} \sin 3 \phi
$$

and

$$
A_{1}=\frac{e Q V_{1}}{2 \pi h}, \quad A_{2}=\frac{e Q V_{2}}{2 \pi h}, \quad A_{3}=\frac{e Q V_{3}}{2 \pi h} .
$$

Here $V_{1}, V_{2}, V_{3}$ are the voltage amplitudes for RF frequencies $h f_{s}, 2 h f_{s}$, and $3 h f_{s}$, respectively. The amplitudes $A_{1}, A_{2}, A_{3}$ are either zero or positive. They are varied slowly during the bunch squeezing.

The equations of motion are

$$
\frac{d \phi}{d t}=\frac{\partial H}{\partial W}=a W
$$

and

$$
\frac{d W}{d t}=-\frac{\partial H}{\partial \phi}=F(\phi)
$$

\section{$3 \quad$ Fixed Points}

Fixed points $\left(\phi_{f}, W_{f}\right)$ of the motion are given by

$$
F\left(\phi_{f}\right)=0, \quad W_{f}=0 .
$$

Since $F(0)=0$ and $F( \pm \pi)=0$ we see that the points $(0,0)$ and $( \pm \pi, 0)$ are fixed points.

Below transition $(a<0)$ a fixed point is stable if

$$
F^{\prime}\left(\phi_{f}\right)>0, \quad U^{\prime \prime}\left(\phi_{f}\right)<0
$$

and unstable if

$$
F^{\prime}\left(\phi_{f}\right)<0, \quad U^{\prime \prime}\left(\phi_{f}\right)>0
$$

where the primes denote differentiation with respect to $\phi$.

Above transition $(a>0)$ a fixed point is stable if

$$
F^{\prime}\left(\phi_{f}\right)<0, \quad U^{\prime \prime}\left(\phi_{f}\right)>0
$$

and unstable if

$$
F^{\prime}\left(\phi_{f}\right)>0, \quad U^{\prime \prime}\left(\phi_{f}\right)<0
$$




\section{The RF Bucket}

Let $H_{u}$ be the value of $H$ at an unstable fixed point $\left(\phi_{u}, 0\right)$. Then

$$
H_{u}=U\left(\phi_{u}\right)
$$

and the equation

$$
H(\phi, W)=H_{u}
$$

can be solved to obtain

$$
W^{2}(\phi)=\frac{2}{a}\left\{U\left(\phi_{u}\right)-U(\phi)\right\} .
$$

The curve $W(\phi)$ is called the separatrix. Differentiating $W^{2}(\phi)$ with respect to $\phi$ we obtain

$$
\frac{d W^{2}}{d \phi}=-\frac{2}{a} U^{\prime}(\phi)
$$

and

$$
\frac{d^{2} W^{2}}{d^{2} \phi}=-\frac{2}{a} U^{\prime \prime}(\phi)
$$

which shows that $W^{2}(\phi)$ reaches a local maximum at each stable fixed point. The area around a stable fixed point and bounded by the separatrix is an RF bucket. The beam contained in the RF bucket is called a bunch. The height $W_{b}$ of the bucket is given by

$$
W_{b}^{2}=\frac{2}{a}\left\{U\left(\phi_{u}\right)-U\left(\phi_{s}\right)\right\}
$$

where $\phi_{s}$ is the stable fixed point phase.

\section{Single-Harmonic Bucket Area}

A single-harmonic bucket is one in which only the fundamental harmonic $h$ is active. In this case we have

$$
F(\phi)=A_{1} \sin \phi
$$

and

$$
\begin{gathered}
U(\phi)=A_{1} \cos \phi \\
U^{\prime}(\phi)=-A_{1} \sin \phi
\end{gathered}
$$




$$
U^{\prime \prime}(\phi)=-A_{1} \cos \phi .
$$

Below transition the stable and unstable fixed point phases are

$$
\phi_{s}=0, \quad \phi_{u}= \pm \pi
$$

and we have

$$
U\left(\phi_{u}\right)=-A_{1} .
$$

The bucket separatrix is then given by

$$
W^{2}(\phi)=-\frac{2 A_{1}}{a}(1+\cos \phi)
$$

and the bucket area is

$$
\mathcal{A}_{1}=2 \int_{-\pi}^{+\pi} W(\phi) d \phi
$$

Using

$$
1+\cos \phi=2 \cos ^{2}(\phi / 2)
$$

we have

$$
\mathcal{A}_{1}=2\left(\frac{4 A_{1}}{-a}\right)^{1 / 2} \int_{-\pi}^{+\pi} \cos (\phi / 2) d \phi
$$

which gives

$$
\mathcal{A}_{1}=16\left(\frac{A_{1}}{-a}\right)^{1 / 2}
$$

Using

$$
a=\left(\frac{h^{2} \omega_{s}^{2} \eta_{s}}{\beta_{s}^{2} E_{s}}\right)=\left(\frac{h^{2} c^{2} \eta_{s}}{R_{s}^{2} E_{s}}\right), \quad A_{1}=\frac{e Q V_{1}}{2 \pi h}
$$

we then have

$$
\mathcal{A}_{1}=8 \frac{R_{s}}{h c}\left\{\frac{2 e Q V_{1} E_{s}}{\pi h\left|\eta_{s}\right|}\right\}^{1 / 2} .
$$

Figure 1 shows a single-harmonic bucket with unstable fixed point phases at \pm 180 degrees. 


\section{Double-Harmonic Bucket Area for $2 A_{2}<A_{1}$}

A double-harmonic bucket is one in which both the fundamental and second harmonics, $h$ and $2 h$, are active. In this case we have

$$
F(\phi)=A_{1} \sin \phi+A_{2} \sin 2 \phi
$$

and

$$
\begin{gathered}
U(\phi)=A_{1} \cos \phi+\frac{1}{2} A_{2} \cos 2 \phi \\
U^{\prime}(\phi)=-A_{1} \sin \phi-A_{2} \sin 2 \phi \\
U^{\prime \prime}(\phi)=-A_{1} \cos \phi-2 A_{2} \cos 2 \phi .
\end{gathered}
$$

Using

$$
\begin{aligned}
& \sin 2 \phi=2 \cos \phi \sin \phi \\
& \cos 2 \phi=2 \cos ^{2} \phi-1
\end{aligned}
$$

gives

$$
\begin{gathered}
U(\phi)=A_{1} \cos \phi+A_{2} \cos ^{2} \phi-\frac{1}{2} A_{2} \\
U^{\prime}(\phi)=-\left(A_{1}+2 A_{2} \cos \phi\right) \sin \phi \\
U^{\prime \prime}(\phi)=-\left(A_{1}+2 A_{2} \cos \phi\right) \cos \phi+2 A_{2} \sin ^{2} \phi .
\end{gathered}
$$

Below transition we again have stable fixed point phase

$$
\phi_{s}=0
$$

and for

$$
0<2 A_{2}<A_{1}
$$

we again have unstable fixed point phases

$$
\phi_{u}= \pm \pi
$$

Thus

$$
U\left(\phi_{u}\right)-U(\phi)=-A_{1}(1+\cos \phi)+A_{2}\left(1-\cos ^{2} \phi\right)
$$

and the bucket area is

$$
\mathcal{A}_{2}=2 \int_{-\pi}^{+\pi} W(\phi) d \phi
$$

where

$$
W^{2}(\phi)=\frac{2}{a}\left\{U\left(\phi_{u}\right)-U(\phi)\right\}
$$


Using

$$
\begin{aligned}
& 1+\cos \phi=2 \cos ^{2}(\phi / 2) \\
& 1-\cos \phi=2 \sin ^{2}(\phi / 2)
\end{aligned}
$$

we have

$$
\begin{gathered}
W^{2}(\phi)=-\frac{2 A_{1}}{a}\left\{2 \cos ^{2}(\phi / 2)-\frac{4 A_{2}}{A_{1}} \cos ^{2}(\phi / 2) \sin ^{2}(\phi / 2)\right\} \\
W^{2}(\phi)=-\frac{4 A_{1}}{a}\left\{1-\frac{2 A_{2}}{A_{1}} \sin ^{2}(\phi / 2)\right\} \cos ^{2}(\phi / 2)
\end{gathered}
$$

and

$$
\mathcal{A}_{2}=2\left(\frac{4 A_{1}}{-a}\right)^{1 / 2} \int_{-\pi}^{+\pi}\left\{1-\frac{2 A_{2}}{A_{1}} \sin ^{2}(\phi / 2)\right\}^{1 / 2} \cos (\phi / 2) d \phi .
$$

Finally, using (41) we have

$$
\mathcal{A}_{2}=\frac{\mathcal{A}_{1}}{4} \int_{-\pi}^{+\pi}\left\{1-\frac{2 A_{2}}{A_{1}} \sin ^{2}(\phi / 2)\right\}^{1 / 2} \cos (\phi / 2) d \phi .
$$

Thus for

$$
2 A_{2}=0
$$

we have

$$
\mathcal{A}_{2}=\mathcal{A}_{1}
$$

(as expected) and as $2 A_{2}$ increases from zero to

$$
2 A_{2}=A_{1}
$$

the bucket area decreases to

$$
\begin{aligned}
& \mathcal{A}_{2}=\frac{\mathcal{A}_{1}}{4} \int_{-\pi}^{+\pi} \cos ^{2}(\phi / 2) d \phi . \\
& \mathcal{A}_{2}=\frac{\mathcal{A}_{1}}{8} \int_{-\pi}^{+\pi}(1+\cos \phi) d \phi
\end{aligned}
$$

which gives

$$
\frac{\mathcal{A}_{2}}{\mathcal{A}_{1}}=\frac{\pi}{4}=0.7853981633974483
$$

Figure 2 shows single and double-harmonic buckets for the cases $2 A_{2}=0$ and $2 A_{2}=A_{1}$, respectively. The bucket area ratio $\mathcal{A}_{2} / \mathcal{A}_{1}$ for the latter case is given by (70). 


\section{Double-Harmonic Bucket Area for $A_{1}<2 A_{2}$}

Consider now the case

$$
0<A_{1}<2 A_{2} .
$$

The fixed point phases (55) then become stable and the unstable fixed point phases are given by

$$
\cos \phi_{u}=-\frac{A_{1}}{2 A_{2}}
$$

Taking the positive phase we have

$$
\pi / 2<\phi_{u}<\pi
$$

Writing

$$
U\left(\phi_{u}\right)-U(\phi)=A_{1}\left(\cos \phi_{u}-\cos \phi\right)+A_{2}\left(\cos ^{2} \phi_{u}-\cos ^{2} \phi\right)
$$

and using

$$
A_{1}=-2 A_{2} \cos \phi_{u}
$$

we have

$$
\begin{gathered}
U\left(\phi_{u}\right)-U(\phi)=-A_{2}\left\{2 \cos \phi_{u}\left(\cos \phi_{u}-\cos \phi\right)+\cos ^{2} \phi-\cos ^{2} \phi_{u}\right\} \\
U\left(\phi_{u}\right)-U(\phi)=-A_{2}\left\{\cos ^{2} \phi-2 \cos \phi_{u} \cos \phi+\cos ^{2} \phi_{u}\right\} \\
U\left(\phi_{u}\right)-U(\phi)=-A_{2}\left(\cos \phi-\cos \phi_{u}\right)^{2}
\end{gathered}
$$

and

$$
W^{2}(\phi)=-\frac{2 A_{2}}{a}\left(\cos \phi-\cos \phi_{u}\right)^{2} .
$$

The bucket area is then

$$
\begin{gathered}
\mathcal{A}_{2}=2\left(\frac{2 A_{2}}{-a}\right)^{1 / 2} \int_{-\phi_{u}}^{\phi_{u}}\left(\cos \phi-\cos \phi_{u}\right) d \phi \\
\mathcal{A}_{2}=4\left(\frac{2 A_{2}}{-a}\right)^{1 / 2}\left(\sin \phi_{u}-\phi_{u} \cos \phi_{u}\right)
\end{gathered}
$$

and using

$$
\frac{2 A_{2}}{-a}=\frac{A_{1}}{a \cos \phi_{u}}
$$


we have

$$
\mathcal{A}_{2}=4\left(\frac{A_{1}}{-a}\right)^{1 / 2}\left(\frac{-1}{\cos \phi_{u}}\right)^{1 / 2}\left(\sin \phi_{u}-\phi_{u} \cos \phi_{u}\right) .
$$

Using single-harmonic bucket area

$$
\mathcal{A}_{1}=16\left(\frac{A_{1}}{-a}\right)^{1 / 2}
$$

we then have

$$
\mathcal{A}_{2}=\frac{\mathcal{A}_{1}}{4}\left(\frac{-1}{\cos \phi_{u}}\right)^{1 / 2}\left(\sin \phi_{u}-\phi_{u} \cos \phi_{u}\right)
$$

Note that for

$$
2 A_{2}=A_{1}
$$

we have

$$
\phi_{u}=\pi
$$

and (85) becomes

$$
\mathcal{A}_{2}=\frac{\pi}{4} \mathcal{A}_{1}
$$

in agreement with (70). In the next section we show that as $2 A_{2}$ increases starting from $2 A_{2}=A_{1}$, the bucket area $\mathcal{A}_{2}$ decreases to a minimum and then begins to increase again.

\section{Minimum Double-Harmonic Bucket Area}

Defining

$$
f\left(\phi_{u}\right)=\left(\frac{-1}{\cos \phi_{u}}\right)^{1 / 2}
$$

and

$$
g\left(\phi_{u}\right)=\sin \phi_{u}-\phi_{u} \cos \phi_{u}
$$

we can write $(85)$ as

$$
\mathcal{A}_{2}=\frac{\mathcal{A}_{1}}{4} f\left(\phi_{u}\right) g\left(\phi_{u}\right) .
$$

To find the minimum bucket area we differentiate $\mathcal{A}_{2}$ with respect to $\phi_{u}$. Thus

$$
\frac{d \mathcal{A}_{2}}{d \phi_{u}}=\frac{\mathcal{A}_{1}}{4}\left\{\frac{d f}{d \phi_{u}} g\left(\phi_{u}\right)+f\left(\phi_{u}\right) \frac{d g}{d \phi_{u}}\right\}
$$




$$
\begin{gathered}
\frac{d f}{d \phi_{u}}=\frac{1}{2} f\left(\phi_{u}\right) \tan \phi_{u}, \quad \frac{d g}{d \phi_{u}}=\phi_{u} \sin \phi_{u} \\
\frac{d \mathcal{A}_{2}}{d \phi_{u}}=\frac{\mathcal{A}_{1}}{4}\left\{\frac{1}{2} g\left(\phi_{u}\right) \tan \phi_{u}+\phi_{u} \sin \phi_{u}\right\} f\left(\phi_{u}\right) \\
\frac{d \mathcal{A}_{2}}{d \phi_{u}}=\frac{\mathcal{A}_{1}}{8}\left\{\frac{g\left(\phi_{u}\right)}{\cos \phi_{u}}+2 \phi_{u}\right\} f\left(\phi_{u}\right) \sin \phi_{u}
\end{gathered}
$$

and

$$
\frac{d \mathcal{A}_{2}}{d \phi_{u}}=\frac{\mathcal{A}_{1}}{8}\left\{\tan \phi_{u}+\phi_{u}\right\} f\left(\phi_{u}\right) \sin \phi_{u}
$$

The bucket area $\mathcal{A}_{2}$ reaches a minimum when

$$
\tan \phi_{u}+\phi_{u}=0
$$

The transcendental equation

$$
\xi+\tan \xi=0
$$

has positive solution

$$
\begin{gathered}
\xi=2.02875783811 \text { (radians) } \\
\xi=116.2392617777881178 \text { (degrees). }
\end{gathered}
$$

We then have

$$
\frac{1}{4} f(\xi) g(\xi)=\frac{1}{2}\left(\frac{\sin \xi}{\sqrt{-\cos \xi}}\right)=0.6744823461666797
$$

and

$$
\frac{-1}{2 \cos \xi}=1.130913167058322
$$

The minimum bucket area is then

$$
\mathcal{A}_{2}(\xi)=0.6744823461666797 \mathcal{A}_{1}
$$

where

$$
\mathcal{A}_{1}=8 \frac{R_{s}}{h c}\left\{\frac{2 e Q V_{1} E_{s}}{\pi h\left|\eta_{s}\right|}\right\}^{1 / 2} .
$$

The corresponding ratio of the harmonic $h$ and $2 h$ voltages is

$$
\frac{V_{2}}{V_{1}}=\frac{A_{2}}{A_{1}}=\frac{-1}{2 \cos \xi}=1.130913167058322 \text {. }
$$


These equations, along with (86) and (88), show that as the amplitude ratio $A_{2} / A_{1}$ goes from $1 / 2$ to the value given by (105), the bucket area ratio $\mathcal{A}_{2} / \mathcal{A}_{1}$ decreases from

$$
\frac{\mathcal{A}_{2}}{\mathcal{A}_{1}}=\frac{\pi}{4}=0.7853981633974483
$$

to the minimum

$$
\frac{\mathcal{A}_{2}}{\mathcal{A}_{1}}=0.6744823461666797 \text {. }
$$

Correspondingly, the unstable fixed point phase $\phi_{u}$ decreases from

$$
\phi_{u}=180 \text { degrees }
$$

to

$$
\phi_{u}=116.2392617777881178 \text { degrees. }
$$

The double-harmonic bucket for which $\mathcal{A}_{2} / \mathcal{A}_{1}$ reaches the minimum (107) is shown in Figure 3.

As the amplitude ratio $A_{2} / A_{1}$ increases starting from the value given by (105), the bucket area ratio $\mathcal{A}_{2} / \mathcal{A}_{1}$ increases monotonically from its minimum value. Correspondingly, the unstable fixed point phase $\phi_{u}$ moves from $\xi$ toward 90 degrees.

Figure 4 shows the double-harmonic bucket for the case in which $A_{2} / A_{1}$ has increased to 3.0. Here the unstable fixed point phase $\phi_{u}$ has moved to 99.594 degrees and the bucket area ratio $\mathcal{A}_{2} / \mathcal{A}_{1}$ has increased to 0.7812 .

A plot of bucket area ratio $\mathcal{A}_{2} / \mathcal{A}_{1}$ versus amplitude ratio $A_{2} / A_{1}$ is shown in Figure 5. This follows from equations (72) and (91).

The corresponding plot of $\phi_{u}$ versus amplitude ratio is shown in Figure 6.

The corresponding plot of bucket area ratio versus $\phi_{u}$ is shown in

Figure 7. 


\section{Triple-Harmonic Bucket Area}

Finally, a triple-harmonic bucket is one in which all three harmonics, $h$, $2 h$, and $3 h$ are active. In this case we have

$$
F(\phi)=A_{1} \sin \phi+A_{2} \sin 2 \phi+A_{3} \sin 3 \phi
$$

and

$$
\begin{gathered}
U(\phi)=A_{1} \cos \phi+\frac{1}{2} A_{2} \cos 2 \phi+\frac{1}{3} A_{3} \cos 3 \phi \\
U^{\prime}(\phi)=-A_{1} \sin \phi-A_{2} \sin 2 \phi-A_{3} \sin 3 \phi \\
U^{\prime \prime}(\phi)=-A_{1} \cos \phi-2 A_{2} \cos 2 \phi-3 A_{3} \cos 3 \phi .
\end{gathered}
$$

Using

$$
\begin{gathered}
\sin 2 \phi=2 \cos \phi \sin \phi \\
\sin 3 \phi=3 \sin \phi-4 \sin ^{3} \phi
\end{gathered}
$$

and

$$
\begin{gathered}
\cos 2 \phi=2 \cos ^{2} \phi-1 \\
\cos 3 \phi=4 \cos ^{3} \phi-3 \cos \phi
\end{gathered}
$$

gives

$$
\begin{gathered}
U(\phi)=A_{1} \cos \phi+\frac{A_{2}}{2}\left(2 \cos ^{2} \phi-1\right)+\frac{A_{3}}{3}\left(4 \cos ^{3} \phi-3 \cos \phi\right) \\
U^{\prime}(\phi)=-\left\{A_{1}+2 A_{2} \cos \phi+A_{3}\left(4 \cos ^{2} \phi-1\right)\right\} \sin \phi \\
U^{\prime \prime}(\phi)=-A_{1} \cos \phi-2 A_{2}\left(2 \cos ^{2} \phi-1\right)-3 A_{3}\left(4 \cos ^{3} \phi-3 \cos \phi\right) .
\end{gathered}
$$

Below transition we again have stable fixed point phase

$$
\phi_{s}=0 \text {. }
$$

We also have fixed point phases given by

$$
A_{1}+2 A_{2} \cos \phi_{f}+A_{3}\left(4 \cos ^{2} \phi_{f}-1\right)=0
$$

which has solutions

$$
\cos \phi_{f}=-\frac{A_{2}}{4 A_{3}} \pm \frac{1}{4 A_{3}} \sqrt{A_{2}^{2}+4 A_{3}\left(A_{3}-A_{1}\right)} .
$$


As shown in the Appendix, the upper sign is associated with the unstable fixed points. Thus we write

$$
\cos \phi_{u}=-\frac{A_{2}}{4 A_{3}}+\frac{1}{4 A_{3}} \sqrt{A_{2}^{2}+4 A_{3}\left(A_{3}-A_{1}\right)}
$$

where $\phi_{u}$ is the unstable fixed point phase.

Introducing the notation

$$
\begin{aligned}
C_{u}=\cos \phi_{u}, & C=\cos \phi \\
\mathcal{U}_{u}=\frac{U\left(\phi_{u}\right)}{A_{1}}, & \mathcal{U}=\frac{U(\phi)}{A_{1}}
\end{aligned}
$$

and

$$
Q=\frac{A_{2}}{A_{1}}, \quad R=\frac{A_{3}}{A_{1}}
$$

we have

$$
\begin{gathered}
\frac{A_{2}}{2 A_{1}}=\frac{Q}{2}, \quad \frac{A_{3}}{3 A_{1}}=\frac{R}{3}, \quad \frac{4 A_{3}}{A_{2}}=\frac{4 R}{Q} \\
\mathcal{U}_{u}=C_{u}+\frac{Q}{2}\left(2 C_{u}^{2}-1\right)+\frac{R}{3}\left(4 C_{u}^{3}-3 C_{u}\right) \\
\mathcal{U}=C+\frac{Q}{2}\left(2 C^{2}-1\right)+\frac{R}{3}\left(4 C^{3}-3 C\right)
\end{gathered}
$$

and

$$
C_{u}=\frac{Q}{4 R}\{\sqrt{1+F}-1\}
$$

where

$$
F=\frac{4 R}{Q^{2}}(R-1)
$$

The separatrix is given by

$$
W^{2}(\phi)=\frac{2 A_{1}}{a}\left(\mathcal{U}_{u}-\mathcal{U}\right)
$$

and the bucket area is

$$
\mathcal{A}_{3}=2\left(\frac{2 A_{1}}{-a}\right)^{1 / 2} \int_{-\phi_{u}}^{\phi_{u}}\left(\mathcal{U}-\mathcal{U}_{u}\right)^{1 / 2} d \phi
$$

where $\phi_{u}$ is the positive phase given by (131) 
Using single-harmonic bucket area

$$
\mathcal{A}_{1}=16\left(\frac{A_{1}}{-a}\right)^{1 / 2}
$$

we then have

$$
\frac{\mathcal{A}_{3}}{\mathcal{A}_{1}}=\frac{\sqrt{2}}{4} \int_{0}^{\phi_{u}}\left(\mathcal{U}-\mathcal{U}_{u}\right)^{1 / 2} d \phi
$$

These equations show that the bucket area ratio $\mathcal{A}_{3} / \mathcal{A}_{1}$ is completely determined by the parameters

$$
Q=\frac{A_{2}}{A_{1}}, \quad R=\frac{A_{3}}{A_{1}} .
$$

Alternatively, one can invert (131) to obtain

$$
R=\frac{1+2 Q C_{u}}{\left(1-2 C_{u}\right)\left(1+2 C_{u}\right)}
$$

which shows that $\mathcal{A}_{3} / \mathcal{A}_{1}$ is completely determined if $\phi_{u}$ and $Q$ are given.

\section{Minimum Triple-Harmonic Bucket Area}

For a given value of amplitude ratio $A_{1} / A_{2}$, one can find the amplitude ratio $R=A_{3} / A_{1}$ for which the bucket area ratio $\mathcal{A}_{3} / \mathcal{A}_{1}$ reaches a minimum. This is done numerically, employing the equations of the previous Section. Figure $\mathbf{8}$ shows the ratio $R$ obtained for several values of $A_{1} / A_{2}$.

The corresponding plots of unstable fixed point phase $\phi_{u}$ and minimum bucket area ratio $\mathcal{A}_{3} / \mathcal{A}_{1}$ versus $A_{1} / A_{2}$ are shown in Figures 9 and 10. The phase $\phi_{u}$ is given by equations (127) and (131). The bucket area ratio is given by (136).

If the single-harmonic bucket area $\mathcal{A}_{1}$ is known, these plots can be used to obtain the minimum triple-harmonic bucket area $\mathcal{A}_{3}$ and the corresponding amplitude ratio $R$ for a given value of $A_{1} / A_{2}$.

As an example, consider the case in which amplitude ratio $A_{1} / A_{2}$ is fixed at $15 / 22$. Using (72) and (91) this gives bucket area ratio $\mathcal{A}_{2} / \mathcal{A}_{1}=0.6826$. Figure 11 shows the corresponding single and double-harmonic buckets.

As the harmonic $3 h$ amplitude is raised from zero, the double-harmonic bucket becomes a triple-harmonic bucket. The area of this bucket will 
decrease from $\mathcal{A}_{3}=\mathcal{A}_{2}$ to a minimum and then begin to increase. The value of $R$ which gives the minimum area can be read off from Figure 8 . There one finds $R=1.994$. The corresponding unstable fixed point phase $\phi_{u}$ obtained from Figure $\mathbf{9}$ is 77.633 degrees. The corresponding bucket area ratio $\mathcal{A}_{3} / \mathcal{A}_{1}$ obtained from Figure $\mathbf{1 0}$ is 0.532 . The minimum-area triple-harmonic bucket is the violet curve shown in Figures $\mathbf{1 2}$ and $\mathbf{1 3}$.

The blue curve in Figure 14 is the triple-harmonic bucket for the case in which $R$ has been raised to $180 / 15$. Figure 15 is a summary showing the triple-harmonic buckets for $R=0,1.994$, and 180/15.

\section{Bunch Merging in AGS}

The most recent setup in Booster and AGS for the delivery of gold ions to RHIC is described in [2]. As reported there, 12 Booster loads each consisting of a single bunch are delivered to AGS per AGS magnetic cycle. The bunch from Booster is the result of a 4 to 2 to 1 bunch merge which is carried out on a flat porch during the Booster magnetic cycle. Each Booster bunch is injected into a harmonic 24 bucket on the AGS injection porch. The bunches are injected into two groups of six adjacent harmonic 24 buckets. In each group the 6 bunches are merged into 3 by bringing on $\mathrm{RF}$ harmonic 12 while reducing harmonic 24 . This is a straightforward 2 to 1 merge in which the two members of a pair of adjacent bunches are merged into one. One ends up with two groups of three adjacent bunches sitting in harmonic 12 buckets. These bunches are accelerated to an intermediate porch for further merging. There, each group of 3 bunches is merged into one by bringing on RF harmonics 8 and 4 and then reducing harmonics 12 and 8. One ends up with 2 bunches, each the result of a 6 to 3 to 1 merge and each sitting in a harmonic 4 bucket.

The nominal revolution frequency of the synchronous particle on the intermediate merging porch is

$$
f_{s}=195.750 \mathrm{kHz}
$$

which gives RF frequency

$$
h f_{s}=783 \mathrm{kHz}
$$

at fundamental harmonic

$$
h=4 .
$$


This is much too low for the standard AGS RF cavities, so a special low-frequency cavity called L10 (after its location in the AGS ring) was made by modifying one of the standard cavities. This was installed in the ring several years ago so that the desired bunch merges could be done. The L10 cavity provides more than enough voltage to hold the merged bunches in their respective harmonic $h$ buckets. However, this single cavity has neither the voltage nor the frequency range to accelerate the bunches to AGS extraction. The merged bunches therefore need to be squeezed so that they can fit into higher harmonic buckets for acceleration.

\section{Bunch Squeezing in AGS}

It is here that the double and triple-harmonic buckets come into their own. In addition to the fundamental RF frequency $h f_{s}$, these buckets require frequencies $2 h f_{s}=1.566 \mathrm{MHz}$ and $3 h f_{s}=2.349 \mathrm{MHz}$. The harmonic $2 h$ frequency is still too low for the standard AGS RF cavity. It is provided by another modified cavity called KL. The harmonic $3 h$ frequency is easily provided by the standard AGS cavities. If the bunch sitting in the harmonic $h$ bucket can be squeezed into a harmonic $3 h$ bucket, it can be accelerated to extraction with the standard AGS cavities.

The squeeze can be done in three steps:

$\underline{\text { First, }}$ with the harmonic $h$ voltage held constant at $V_{1}$, the harmonic $2 h$ voltage is raised adiabatically from zero to $V_{2}$. For

$$
0 \leq 2 V_{2}<V_{1}
$$

this produces a double-harmonic bucket with area $\mathcal{A}_{2}$ given by (64). For

$$
0<V_{1}<2 V_{2}
$$

the bucket area is given by (85). As long as the longitudinal emittance of the merged bunch is less than the minimum double-harmonic bucket area given by (103) and (104), the double-harmonic bucket is guaranteed to hold the bunch as the harmonic $2 h$ voltage is raised from zero.

Next, with the harmonic $h$ and $2 h$ voltages held constant at $V_{1}$ and $V_{2}$ respectively, the harmonic $3 h$ voltage is raised adiabatically from zero to $V_{3}$. This produces a triple-harmonic bucket with area $\mathcal{A}_{3}$ given by (136). Again, as long as the longitudinal emittance of the bunch is less than the 
minimum triple-harmonic bucket area, the triple-harmonic bucket will hold the bunch.

Finally, with the harmonic $3 h$ voltage held constant at $V_{3}$, the harmonic $h$ $\overline{\text { and } 2 h}$ voltages are lowered adiabatically to zero. Provided $V_{3}$ is sufficiently large, this leaves the merged bunch sitting in a harmonic $3 h$ bucket ready to be accelerated. The area of the bucket is

$$
A_{3 h}=8 \frac{R_{s}}{3 h c}\left\{\frac{2 e Q V_{3} E_{s}}{\pi 3 h\left|\eta_{s}\right|}\right\}^{1 / 2}
$$

and in terms of the single-harmonic bucket area

$$
\mathcal{A}_{1}=8 \frac{R_{s}}{h c}\left\{\frac{2 e Q V_{1} E_{s}}{\pi h\left|\eta_{s}\right|}\right\}^{1 / 2}
$$

we have

$$
A_{3 h}=\frac{\mathcal{A}_{1}}{3 \sqrt{3}} \sqrt{V_{3} / V_{1}}
$$

For Au77+ ions circulating in AGS at nominal radius

$$
R_{s}=128.4526 \mathrm{~m}
$$

and with revolution frequency (139), the bucket area (145) for voltage

$$
V_{0}=15 \mathrm{kV} \text { perturn }
$$

is

$$
\mathcal{A}_{0}=1.02944 \mathrm{eV} \text { s per nucleon. }
$$

Here we have used Au77+ mass-energy equivalent

$$
m c^{2}=183.434174442 \mathrm{GeV}
$$

and transition gamma

$$
\gamma_{t}=8.5
$$

The single-harmonic bucket area $\mathcal{A}_{1}$ for any voltage $V_{1}$ is then

$$
\mathcal{A}_{1}=\mathcal{A}_{0} \sqrt{V_{1} / V_{0}} .
$$

The corresponding double and triple-harmonic bucket areas $\mathcal{A}_{2}$ and $\mathcal{A}_{3}$ can be obtained from bucket area ratios $\mathcal{A}_{2} / \mathcal{A}_{1}$ and $\mathcal{A}_{3} / \mathcal{A}_{1}$. 
Using (152) in (146) gives

$$
A_{3 h}=\frac{\mathcal{A}_{0}}{3 \sqrt{3}} \sqrt{V_{3} / V_{0}} .
$$

For voltages $V_{3}=180,189,198,207,216,225$, and $234 \mathrm{kV}$ one then

obtains harmonic $3 h$ bucket areas $A_{3 h}=0.686,0.703,0.720,0.736,0.752$,

0.767 , and $0.782 \mathrm{eV}$ s per nucleon. These are upper limits on the

longitudinal emittance of a merged and squeezed Au77+ bunch sitting in a harmonic $3 h=12$ bucket on the merging porch prior to acceleration.

\section{Acceptance of the Squeeze}

Figure 16 shows the double-harmonic bucket area $\mathcal{A}_{2}$ obtained for various values of $V_{1}$ and $V_{2}$. The brown, orange, violet, blue, and magenta curves give $\mathcal{A}_{2}$ as a function of $V_{2}$ with $V_{1}$ fixed at $12,15,18,21$, and $22 \mathrm{kV}$, respectively. These curves reach minimum double-harmonic bucket areas $\mathcal{A}_{2}=0.621,0.694,0.761,0.822$, and $0.841 \mathrm{eV}$ s per nucleon, respectively. The corresponding voltages $V_{2}$ are $13.6,17.0,20.4,23.7$, and $24.9 \mathrm{kV}$. If, for a given $V_{1}$, the rising second harmonic voltage $V_{2}$ goes through the value for which a minimum is reached, the longitudinal emittance of the merged Au77+ bunch must be less than that minimum if the bunch is to be held in the double-harmonic bucket. This minimum is the acceptance of the double-harmonic portion of the squeeze. If, for example, $V_{1}$ is fixed at $15 \mathrm{kV}$ and the rising voltage $V_{2}$ goes through $17.0 \mathrm{kV}$, then the longitudinal emittance of the merged bunch must be less than $0.694 \mathrm{eV}$ s per nucleon.

Figure 17 shows the triple-harmonic bucket area $\mathcal{A}_{3}$ obtained for various values of $V_{1}$ and $V_{3}$ with $\underline{V_{2}}$ fixed at $22 \mathrm{kV}$. The brown, orange, violet, blue, and magenta curves give $\mathcal{A}_{3}$ as a function of $V_{3}$ with $V_{1}$ fixed at 12 , $15,18,21$, and $22 \mathrm{kV}$, respectively. These curves reach minimum triple-harmonic bucket areas $\mathcal{A}_{3}=0.524,0.548,0.571,0.593$, and 0.600 eV s per nucleon, respectively. The corresponding voltages $V_{3}$ are 26.2, $29.9,33.7,37.4$, and $38.7 \mathrm{kV}$. If, for a given $V_{1}$, the rising third harmonic voltage $V_{3}$ goes through the value for which a minimum is reached, the longitudinal emittance of the merged Au77+ bunch must be less than that minimum if the bunch is to be held in the triple-harmonic bucket. This minimum is the acceptance of the triple-harmonic portion of the squeeze. If, for example, $V_{1}$ is fixed at $15 \mathrm{kV}$ and the rising voltage $V_{3}$ goes through $29.9 \mathrm{kV}$, then the longitudinal emittance of the merged bunch must be less than $0.548 \mathrm{eV} \mathrm{s}$ per nucleon. 
The minimum bucket areas $0.524,0.548,0.571 \mathrm{eV}$ s per nucleon obtained here for $V_{1}=12,15,18 \mathrm{kV}$, respectively, are in good agreement with the corresponding acceptances $0.526,0.550,0.574$ obtained by simulation in [2].

Figure 18 shows the triple-harmonic bucket area $\mathcal{A}_{3}$ obtained for various values of $V_{1}$ and $V_{3}$ with $\underline{V_{2}}$ fixed at $24 \mathrm{kV}$. The brown, orange, violet, blue, and magenta curves give $\mathcal{A}_{3}$ as a function of $V_{3}$ with $V_{1}$ fixed at 12 , $15,18,21$, and $22 \mathrm{kV}$, respectively. These curves reach minimum triple-harmonic bucket areas $\mathcal{A}_{3}=0.539,0.562,0.585,0.606$, and 0.613 $\mathrm{eV}$ s per nucleon, respectively. The corresponding voltages $V_{3}$ are 27.2, $30.9,34.7,38.4$, and $39.7 \mathrm{kV}$. As before, if, for a given $V_{1}$, the rising voltage $V_{3}$ goes through the value for which a minimum is reached, the longitudinal emittance of the merged Au77+ bunch must be less than that minimum if the bunch is to be held in the triple-harmonic bucket. In this case, if $V_{1}$ is fixed at $15 \mathrm{kV}$ then the longitudinal emittance of the merged bunch must be less than $0.562 \mathrm{eV}$ s per nucleon.

Finally, Figure 19 shows the triple-harmonic bucket area $\mathcal{A}_{3}$ obtained for various values of $V_{1}$ and $V_{3}$ with $V_{2}$ fixed at $20 \mathrm{kV}$. The brown, orange, violet, blue, and magenta curves give $\mathcal{A}_{3}$ as a function of $V_{3}$ with $V_{1}$ fixed at $12,15,18,21$, and $22 \mathrm{kV}$, respectively. These curves pass through minimum triple-harmonic bucket areas $\mathcal{A}_{3}=0.509,0.534,0.557,0.580$, and $0.587 \mathrm{eV} \mathrm{s}$ per nucleon, respectively. The corresponding voltages $V_{3}$ are $25.1,28.9,32.7,36.4$, and $37.7 \mathrm{kV}$. As before, if, for a given $V_{1}$, the rising voltage $V_{3}$ goes through the value for which a minimum is reached, the longitudinal emittance of the merged Au77+ bunch must be less than that minimum if the bunch is to be held in the triple-harmonic bucket. In this case, if $V_{1}$ is fixed at $15 \mathrm{kV}$ then the longitudinal emittance of the merged bunch must be less than $0.534 \mathrm{eV}$ s per nucleon.

Just before squeezing, the longitudinal emittance of a merged Au77+ bunch in AGS can be as large as $0.6 \mathrm{eV}$ s per nucleon in practice. The above results show that in order to hold such a bunch in a single bucket during squeezing, one needs at least $22 \mathrm{kV}$ for both the L10 (harmonic $h$ ) and KL (harmonic $2 h$ ) RF cavities.

If the harmonic $h$ and $2 h$ voltages are instead 15 and $22 \mathrm{kV}$, respectively, then the acceptance is reduced to $0.548 \mathrm{eV} \mathrm{s}$ per nucleon. 


\section{Appendix}

Here we have a closer look at the triple-harmonic fixed points. It is assumed that we are operating on a porch below transition.

For the triple-harmonic bucket we have

$$
F(\phi)=A_{1} \sin \phi+A_{2} \sin 2 \phi+A_{3} \sin 3 \phi
$$

and

$$
\begin{gathered}
U(\phi)=A_{1} \cos \phi+\frac{1}{2} A_{2} \cos 2 \phi+\frac{1}{3} A_{3} \cos 3 \phi \\
U^{\prime}(\phi)=-A_{1} \sin \phi-A_{2} \sin 2 \phi-A_{3} \sin 3 \phi \\
U^{\prime \prime}(\phi)=-A_{1} \cos \phi-2 A_{2} \cos 2 \phi-3 A_{3} \cos 3 \phi .
\end{gathered}
$$

Using

$$
\begin{gathered}
\sin 2 \phi=2 \cos \phi \sin \phi \\
\sin 3 \phi=3 \sin \phi-4 \sin ^{3} \phi
\end{gathered}
$$

and

$$
\begin{gathered}
\cos 2 \phi=2 \cos ^{2} \phi-1 \\
\cos 3 \phi=4 \cos ^{3} \phi-3 \cos \phi
\end{gathered}
$$

gives

$$
\begin{gathered}
U(\phi)=A_{1} \cos \phi+\frac{A_{2}}{2}\left(2 \cos ^{2} \phi-1\right)+\frac{A_{3}}{3}\left(4 \cos ^{3} \phi-3 \cos \phi\right) \\
U^{\prime}(\phi)=-\left\{A_{1}+2 A_{2} \cos \phi+A_{3}\left(4 \cos ^{2} \phi-1\right)\right\} \sin \phi \\
U^{\prime \prime}(\phi)=-A_{1} \cos \phi-2 A_{2}\left(2 \cos ^{2} \phi-1\right)-3 A_{3}\left(4 \cos ^{3} \phi-3 \cos \phi\right) .
\end{gathered}
$$

Fixed point phases $\phi_{f}$ are given by

$$
U^{\prime}\left(\phi_{f}\right)=0 .
$$

The fixed point is stable (below transition) if

$$
U^{\prime \prime}\left(\phi_{f}\right)<0
$$

and unstable if

$$
U^{\prime \prime}\left(\phi_{f}\right)>0
$$




\subsection{Notation}

Introducing the notation

$$
\begin{gathered}
\mathcal{U}_{a}=\frac{U\left(\phi_{a}\right)}{A_{1}}, \quad \mathcal{U}_{a}^{\prime}=\frac{U^{\prime}\left(\phi_{a}\right)}{A_{1}}, \quad \mathcal{U}_{a}^{\prime \prime}=\frac{U^{\prime \prime}\left(\phi_{a}\right)}{A_{1}} \\
Q=\frac{A_{2}}{A_{1}}, \quad R=\frac{A_{3}}{A_{1}}
\end{gathered}
$$

and

$$
C_{a}=\cos \phi_{a}, \quad S_{a}=\sin \phi_{a}
$$

we have

$$
\frac{A_{2}}{2 A_{1}}=\frac{Q}{2}, \quad \frac{A_{3}}{3 A_{1}}=\frac{R}{3}, \quad \frac{4 A_{3}}{A_{2}}=\frac{4 R}{Q}
$$

and

$$
\begin{gathered}
\mathcal{U}_{a}=C_{a}+\frac{Q}{2}\left(2 C_{a}^{2}-1\right)+\frac{R}{3}\left(4 C_{a}^{3}-3 C_{a}\right) \\
\mathcal{U}_{a}^{\prime}=-\left\{1+2 Q C_{a}+R\left(4 C_{a}^{2}-1\right)\right\} S_{a} \\
\mathcal{U}_{a}^{\prime \prime}=-C_{a}-2 Q\left(2 C_{a}^{2}-1\right)-3 R\left(4 C_{a}^{3}-3 C_{a}\right) .
\end{gathered}
$$

\subsection{Fixed point phases $\phi_{f}=0$ and $\phi_{f}= \pm \pi$}

For

$$
\phi_{f}=0
$$

we have

$$
\mathcal{U}_{f}^{\prime}=0, \quad \mathcal{U}_{f}^{\prime \prime}=-1-2 Q-3 R
$$

which shows that we have stable fixed point phase

$$
\phi_{s}=0 .
$$

For

$$
\phi_{f}= \pm \pi
$$

we have

$$
\mathcal{U}_{f}^{\prime}=0, \quad \mathcal{U}_{f}^{\prime \prime}=1-2 Q+3 R
$$

which shows that for

$$
1+3 R<2 Q
$$


we have stable fixed point phases

$$
\phi_{s}= \pm \pi
$$

and for

$$
1+3 R>2 Q
$$

these become unstable fixed point phases

$$
\phi_{u}= \pm \pi
$$

\subsection{Additional fixed points I}

Equation (173) shows that we have additional fixed point phases given by

$$
1+2 Q C_{f}+R\left(4 C_{f}^{2}-1\right)=0 .
$$

For $\underline{R=0}$ this gives

$$
1+2 Q C_{f}=0
$$

and

$$
C_{f}=-\frac{1}{2 Q}=-\frac{A_{1}}{2 A_{2}}
$$

in agreement with (72). Here, in order to have $\left|C_{f}\right| \leq 1$, we must have

$$
Q \geq \frac{1}{2}
$$

Thus we have

$$
\begin{gathered}
\mathcal{U}_{f}^{\prime \prime}=-C_{f}-2 Q\left(2 C_{f}^{2}-1\right) \\
\mathcal{U}_{f}^{\prime \prime}=\frac{1}{2 Q}-2 Q\left(\frac{1}{2 Q^{2}}-1\right) \\
\mathcal{U}_{f}^{\prime \prime}=\frac{1}{2 Q}\left(4 Q^{2}-1\right) \geq 0
\end{gathered}
$$

and we see that the fixed point is unstable, in agreement with (72).

On the other hand, for $R \gg Q$, equation (184) gives

$$
C_{f}= \pm \frac{1}{2}
$$

and we have

$$
\mathcal{U}_{f}^{\prime \prime}=-3 R C_{f}\left(4 C_{f}^{2}-3\right)= \pm 3 R .
$$


This gives unstable fixed point phase

$$
\phi_{u}=\frac{\pi}{3}
$$

for the upper sign in (192), and stable fixed point phase

$$
\phi_{s}=\frac{2 \pi}{3}
$$

for the lower sign. This is just as expected for a harmonic $3 h$ bucket.

\subsection{Additional fixed points II}

Rewriting (184) as

$$
4 R C_{f}^{2}+2 Q C_{f}+1-R=0
$$

we see that

$$
C_{f}=-\frac{Q}{4 R} \pm \frac{1}{4 R} \sqrt{Q^{2}+4 R(R-1)} .
$$

Here the expression under the square root must be non-negative, so we must have

$$
4 R(R-1) \geq-Q^{2}
$$

which can be written as

$$
\left(R-\frac{1}{2}\right)^{2} \geq \frac{1-Q^{2}}{4} .
$$

Equation (197) is obviously satisfied for all $Q$ if

$$
R \geq 1
$$

and (198) is obviously satisfied for all $R$ if

$$
Q^{2} \geq 1 \text {. }
$$

On the other hand, if

$$
Q^{2}<1
$$

then (198) is satisfied unless

$$
\frac{1}{2}-\sqrt{G}<R<\frac{1}{2}+\sqrt{G}
$$

where

$$
G=\frac{1-Q^{2}}{4} .
$$

In practice one typically has $V_{2} \geq V_{1}$ when the harmonic $3 h$ voltage is brought on, so for our purposes here we simply assume that

$$
Q \geq 1 \text {. }
$$




\subsection{Additional fixed points III}

For the additional fixed points we have

$$
4 R C_{f}^{2}+2 Q C_{f}+1-R=0 .
$$

Multiplying this by $3 C_{f}$ and adding the result to the right-hand side of

$$
\mathcal{U}_{f}^{\prime \prime}=-C_{f}-2 Q\left(2 C_{f}^{2}-1\right)-3 R\left(4 C_{f}^{3}-3 C_{f}\right)
$$

gives

$$
\mathcal{U}_{f}^{\prime \prime}=2 Q C_{f}^{2}+2(3 R+1) C_{f}+2 Q .
$$

We can then write

$$
\mathcal{U}_{f}^{\prime \prime}=2 Q K_{f}
$$

where

$$
K_{f}=C_{f}^{2}+2\left(\frac{3 R+1}{2 Q}\right) C_{f}+1 .
$$

Thus the fixed points are stable if

$$
K_{f}<0
$$

and unstable if

$$
K_{f}>0 .
$$

In the following sections we show that the unstable fixed points are associated with the upper $(+)$ sign in (196). Accordingly we define

$$
C_{u}=-\frac{Q}{4 R}+\frac{1}{4 R} \sqrt{Q^{2}+4 R(R-1)}
$$

and

$$
K_{u}=C_{u}^{2}+2\left(\frac{3 R+1}{2 Q}\right) C_{u}+1
$$

where

$$
C_{u}=\cos \phi_{u}
$$

and $\phi_{u}$ is the unstable fixed point phase. 


\subsection{Additional fixed point, case $R \geq 1$}

Recalling the equations

$$
C_{u}=-\frac{Q}{4 R}+\frac{1}{4 R} \sqrt{Q^{2}+4 R(R-1)}
$$

and

$$
K_{u}=C_{u}^{2}+2\left(\frac{3 R+1}{2 Q}\right) C_{u}+1
$$

we see that for

$$
R \geq 1
$$

we have

$$
C_{u} \geq 0
$$

and

$$
K_{u} \geq 1 \text {. }
$$

This gives an unstable fixed point phase.

\subsection{Additional fixed point, case $0<R<1$}

Introducing the notation

$$
\mathcal{C}=\frac{4 R}{Q} C_{u}, \quad \mathcal{K}=\frac{16 R^{2}}{Q^{2}} K_{u}
$$

we have

$$
\mathcal{K}=\mathcal{C}^{2}+\frac{4 R}{Q^{2}}(3 R+1) \mathcal{C}+\frac{16 R^{2}}{Q^{2}}
$$

and

$$
\mathcal{C}=-1+\sqrt{1+F}
$$

where

$$
F=\frac{4 R(R-1)}{Q^{2}}=\frac{4}{Q^{2}}\left\{\left(R-\frac{1}{2}\right)^{2}-\frac{1}{4}\right\} .
$$

Thus

$$
\begin{gathered}
\mathcal{K}=\mathcal{C}^{2}+\frac{12 R^{2}}{Q^{2}} \sqrt{1+F}+\frac{4 R^{2}}{Q^{2}}-\frac{4 R}{Q^{2}}+\frac{4 R}{Q^{2}} \sqrt{1+F} \\
\mathcal{K}=\mathcal{C}^{2}+\frac{12 R^{2}}{Q^{2}} \sqrt{1+F}+F+\frac{4 R}{Q^{2}} \sqrt{1+F}
\end{gathered}
$$


and using

$$
\mathcal{C}^{2}=1-2 \sqrt{1+F}+1+F
$$

we have

$$
\mathcal{K}=2(1+F)-2 \sqrt{1+F}+\frac{12 R^{2}}{Q^{2}} \sqrt{1+F}+\frac{4 R}{Q^{2}} \sqrt{1+F} .
$$

We want to show that $\mathcal{K}>0$. To do so it is helpful to factor $\sqrt{1+F}$ out of equation (227). Thus we write

$$
\mathcal{K}=\mathcal{H} \sqrt{1+F}
$$

where

$$
\mathcal{H}=2 \sqrt{1+F}-2+\frac{4 R}{Q^{2}}(3 R+1) .
$$

Then for

$$
0<R \ll 1
$$

we have, to second order in $R$,

$$
\begin{gathered}
\sqrt{1+F}=1+\frac{F}{2}-\frac{F^{2}}{8} \\
F=\frac{4}{Q^{2}}\left(R^{2}-R\right), \quad F^{2}=\frac{16 R^{2}}{Q^{4}} \\
\sqrt{1+F}=1-\frac{2 R}{Q^{2}}+\frac{2 R^{2}}{Q^{2}}\left(1-\frac{1}{Q^{2}}\right) \\
2 \sqrt{1+F}-2=-\frac{4 R}{Q^{2}}+\frac{4 R^{2}}{Q^{2}}\left(1-\frac{1}{Q^{2}}\right)
\end{gathered}
$$

and

$$
\mathcal{H}=\frac{4 R^{2}}{Q^{2}}\left(4-\frac{1}{Q^{2}}\right)>0
$$

For

$$
R=\frac{1}{2}
$$

we have

$$
F=-\frac{1}{Q^{2}}
$$

and

$$
\mathcal{H}=\frac{5}{Q^{2}}-2+2\left(1-\frac{1}{Q^{2}}\right)^{1 / 2}>0 .
$$


For

$$
R=1
$$

we have

$$
F=0, \quad \mathcal{H}=\frac{16}{Q^{2}}>0 .
$$

Thus for

$$
0<R<1
$$

we conclude

$$
\mathcal{H}>0, \quad \mathcal{K}>0
$$

and we again have an unstable fixed point.

\section{References}

[1] C.J. Gardner, et al, "Operation of the RHIC Injector Chain with Ions from EBIS", Proceedings of IPAC2015, Richmond, Virginia, USA, pp. 3804-3807.

[2] C.J. Gardner, "Simulation of 6 to 3 to 1 merge and squeeze of Au77+ bunches in AGS", C-A/AP/Note 563, May 2016. 


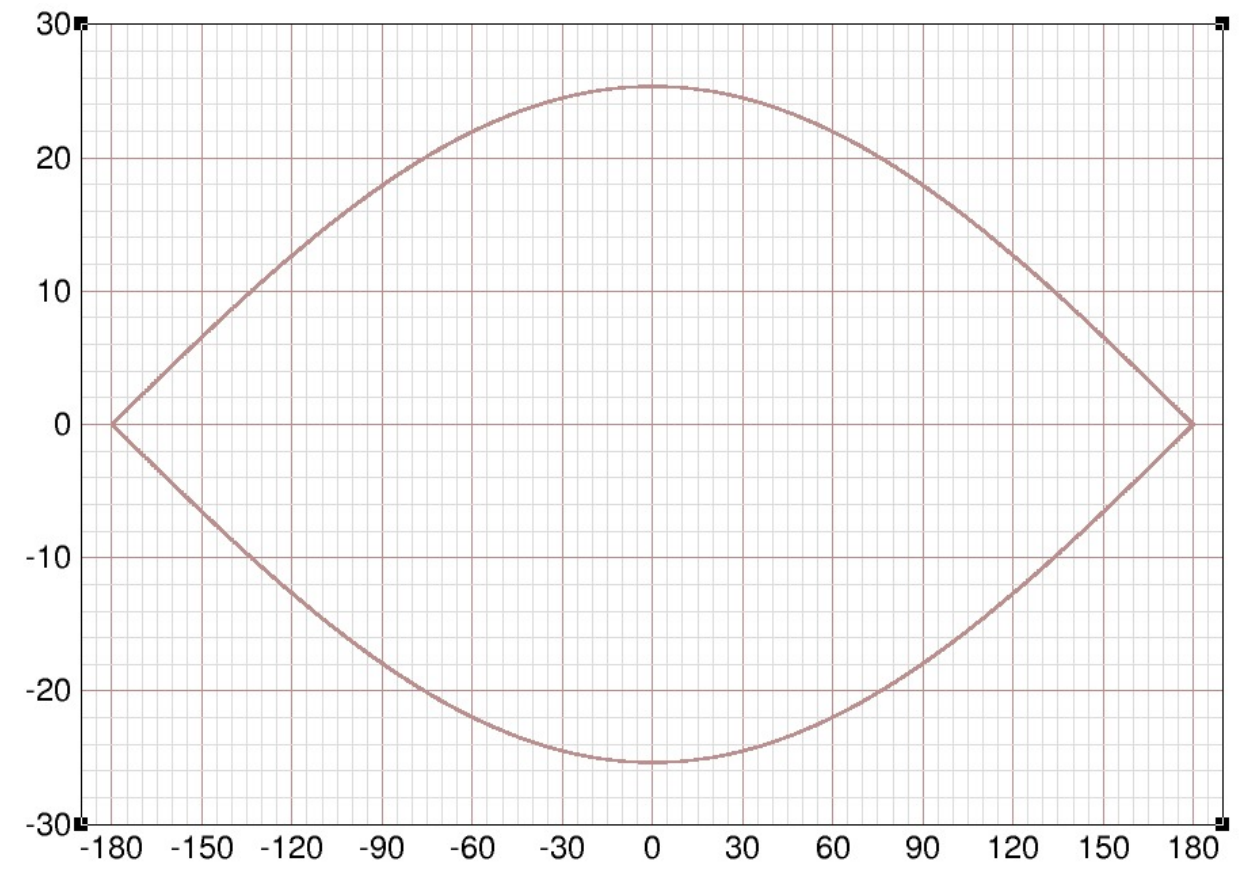

Figure 1: Single-harmonic RF bucket. This is a bucket for which only the fundamental harmonic $h$ is active. The horizontal axis gives the RF phase in degrees. The vertical axis gives the energy deviation $E-E_{s}$ divided by $2 \pi h f_{s}$. We use calligraphic $\mathcal{A}_{1}$ to denote the area of the bucket. The unstable fixed point phases are \pm 180 degrees. 


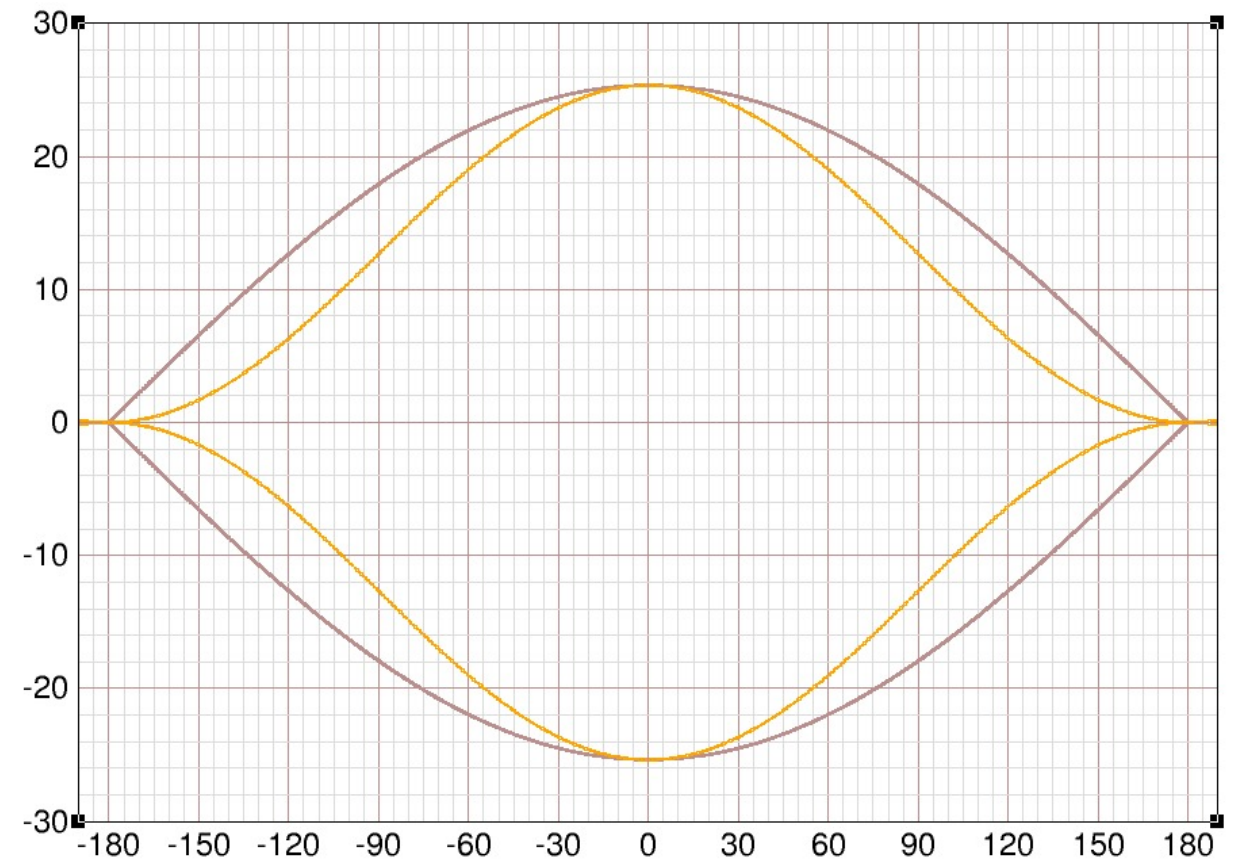

Figure 2: Single and double-harmonic buckets. These are the brown and orange curves respectively. The double-harmonic bucket is one in which both the fundamental and second harmonics, $h$ and $2 h$, are active. We use calligraphic $\mathcal{A}_{2}$ to denote its area. Here the ratio of the second harmonic voltage to that of the fundamental harmonic is $V_{2} / V_{1}=1 / 2$. This gives bucket area ratio $\mathcal{A}_{2} / \mathcal{A}_{1}=\pi / 4$ as shown in Section 6 . The unstable fixed point phases are again \pm 180 degrees. 


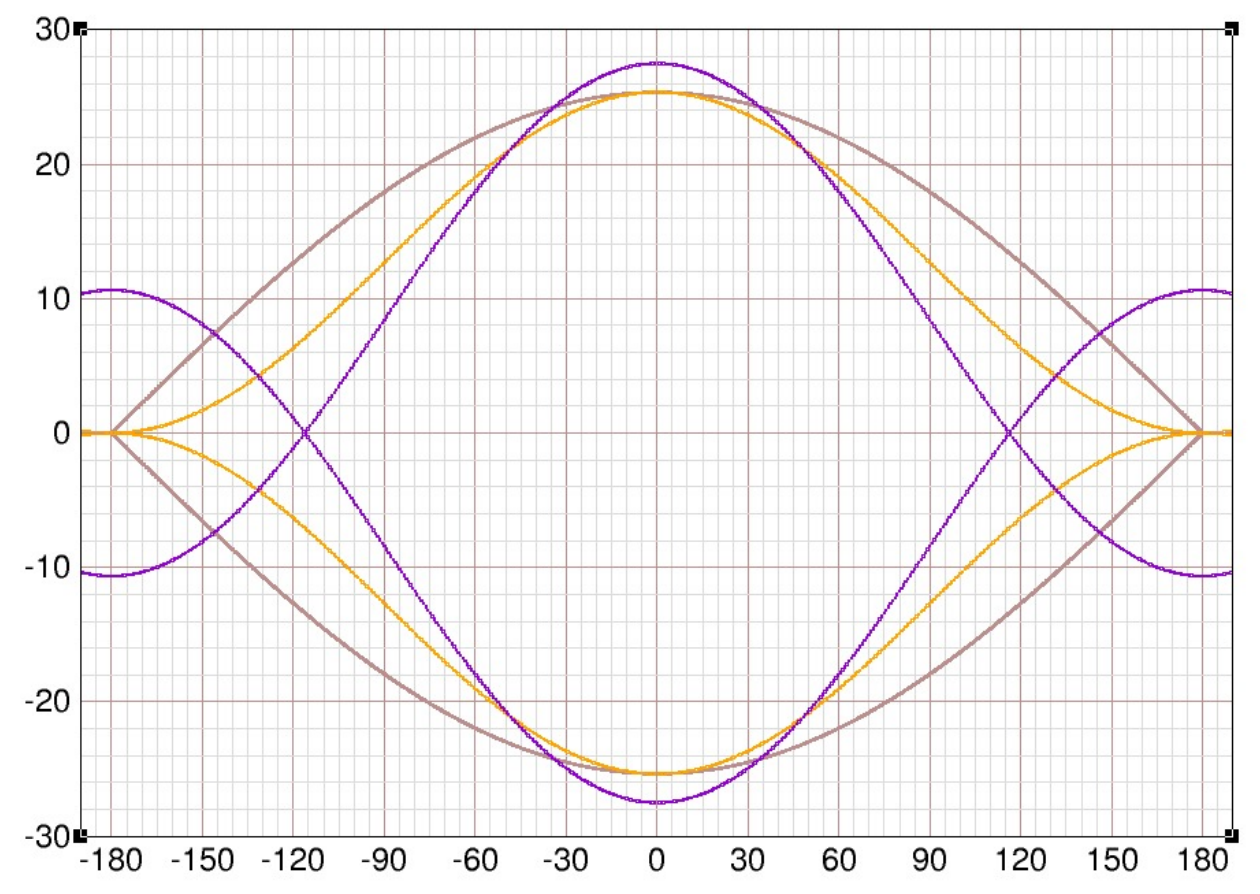

Figure 3: Here the brown and orange curves are the same as those in Figure 2. The violet curve is the double-harmonic bucket for the case in which the bucket area ratio $\mathcal{A}_{2} / \mathcal{A}_{1}$ reaches its minimum (107). This is approximately 0.67 . The voltage ratio $V_{2} / V_{1}$, given by (105), is approximately 1.13 . The unstable fixed point phases, given by (109), are approximately \pm 116 degrees. 


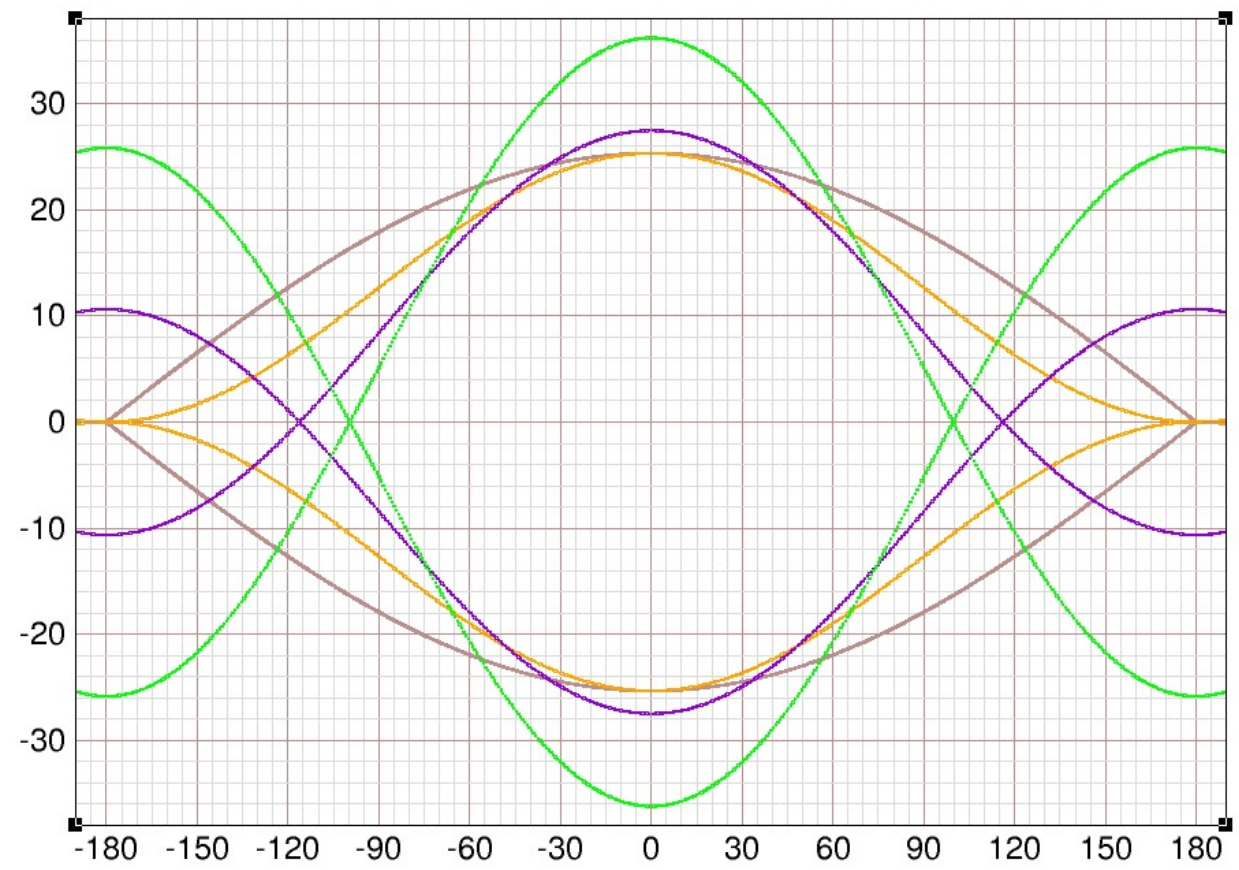

Figure 4: Here the brown, orange, and violet curves are the same as those in Figure 3. The voltage ratios $V_{2} / V_{1}$ for these curves are $0,0.5$, and 1.13 , respectively. The green curve is the double-harmonic bucket for the case in which $V_{2} / V_{1}$ has increased to 3.0. Here the unstable fixed point phase $\phi_{u}$ has moved to 99.594 degrees and the bucket area ratio $\mathcal{A}_{2} / \mathcal{A}_{1}$ has increased to 0.7812 . (Note that since $A_{2} / A_{1}=V_{2} / V_{1}$ we use amplitude ratio and voltage ratio interchangeably.) 


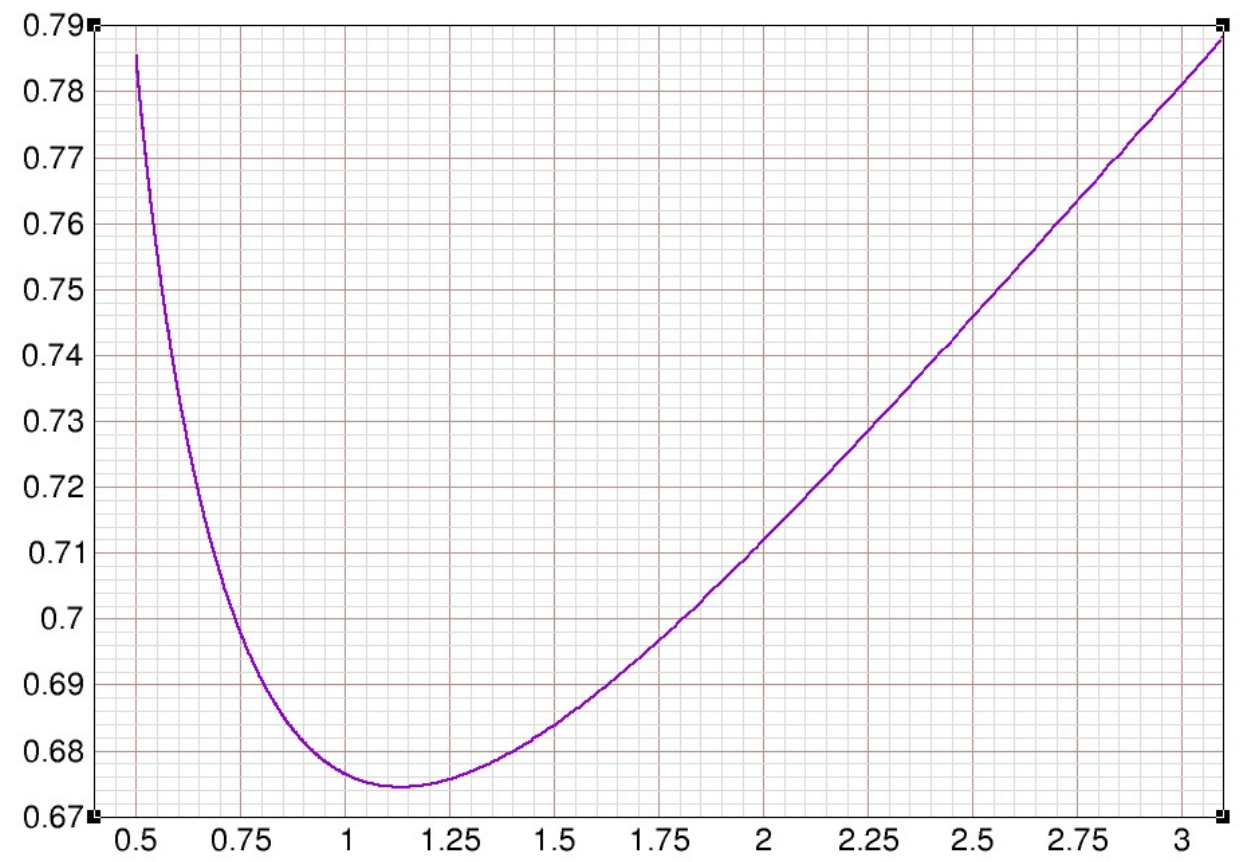

Figure 5: Bucket area ratio $\mathcal{A}_{2} / \mathcal{A}_{1}$ versus voltage ratio $V_{2} / V_{1}$. This follows from equations (72) and (91). The bucket area ratio reaches its minimum (107) at the voltage ratio given by (105). (Note, again, that since $A_{2} / A_{1}=$ $V_{2} / V_{1}$ we use amplitude ratio and voltage ratio interchangeably.) 


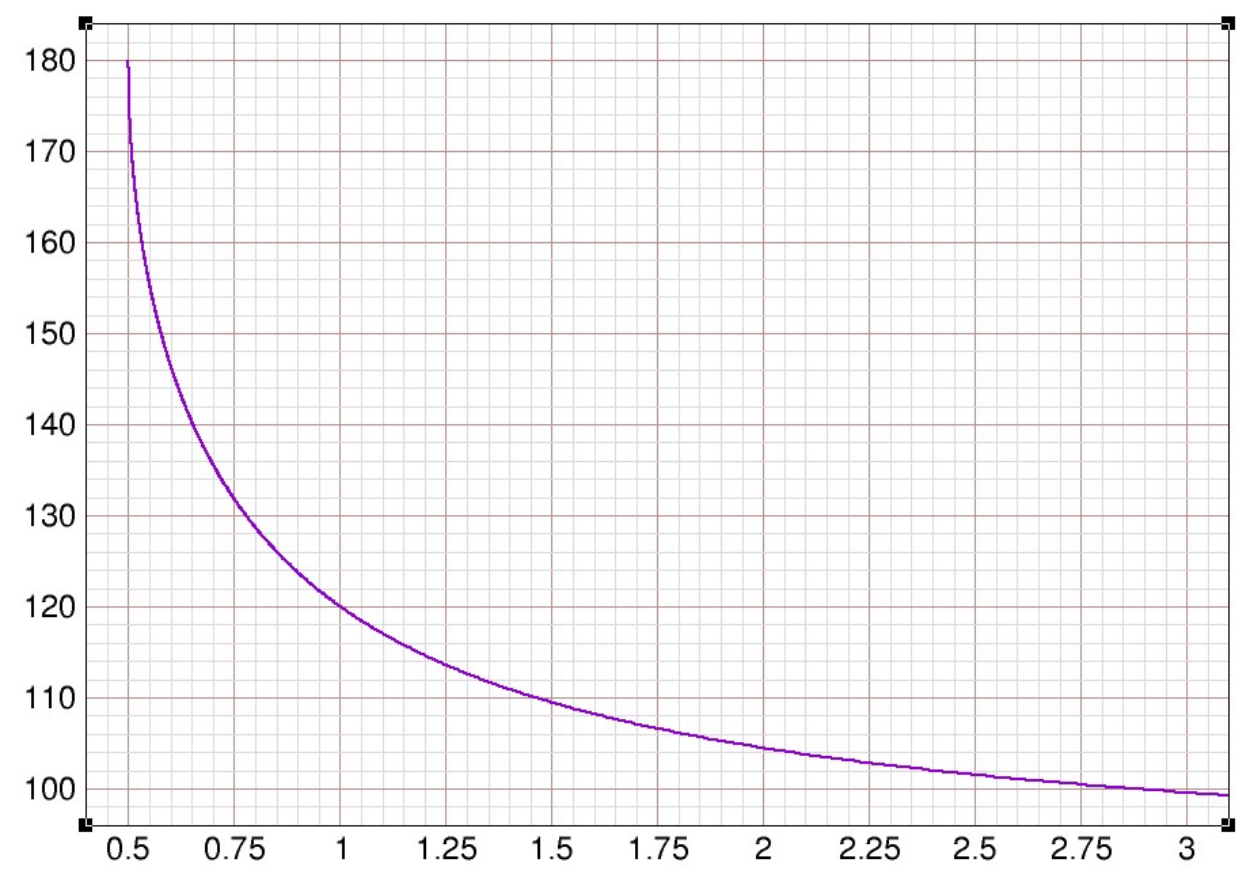

Figure 6: Double-harmonic bucket unstable fixed point phase $\phi_{u}$ versus voltage ratio $V_{2} / V_{1}$. This follows from equation (72). 


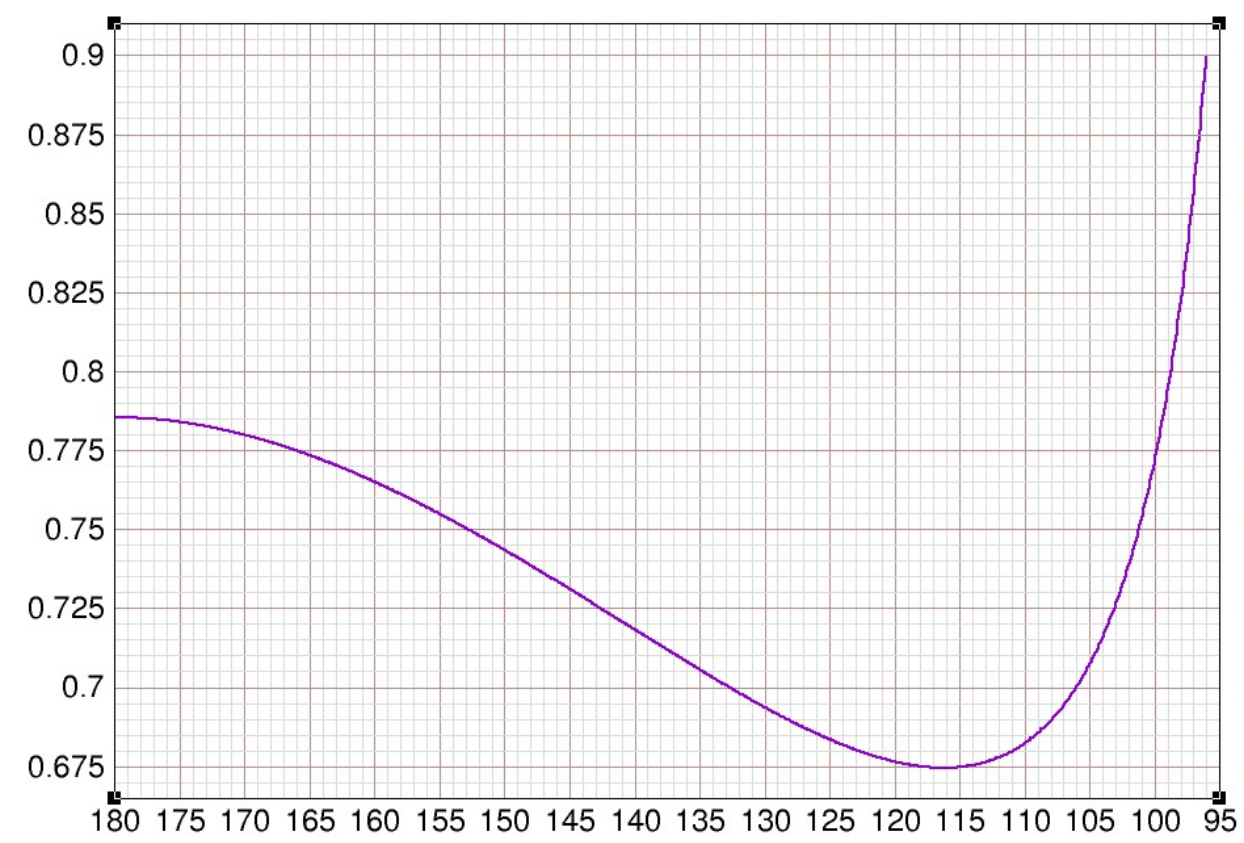

Figure 7: Bucket area ratio $\mathcal{A}_{2} / \mathcal{A}_{1}$ versus unstable fixed point phase $\phi_{u}$. This follows from equation (91). The unstable fixed point phase for which $\mathcal{A}_{2} / \mathcal{A}_{1}$ reaches its minimum is given by (109). This is approximately 116 degrees. 


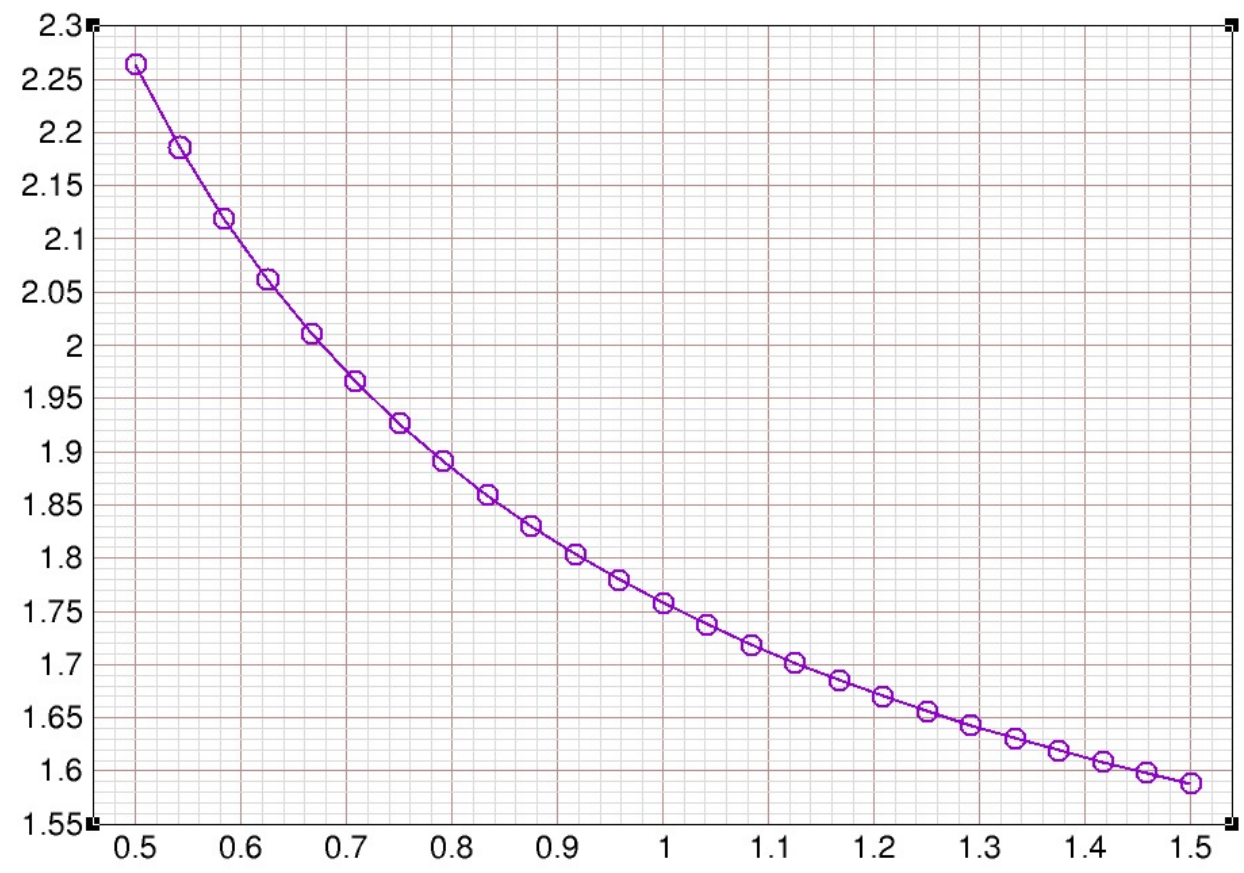

Figure 8: The triple-harmonic bucket is one in which the fundamental, second, and third harmonics, $h, 2 h$, and $3 h$ are all active. We use calligraphic $\mathcal{A}_{3}$ to denote its area. Here the amplitude ratio $R=A_{3} / A_{1}$ (vertical axis) for which bucket area ratio $\mathcal{A}_{3} / \mathcal{A}_{1}$ reaches a minimum has been calculated for several values of the amplitude ratio $A_{1} / A_{2}$ (horizontal axis). These data are indicated by the violet circles. The violet curve connects the circles. 


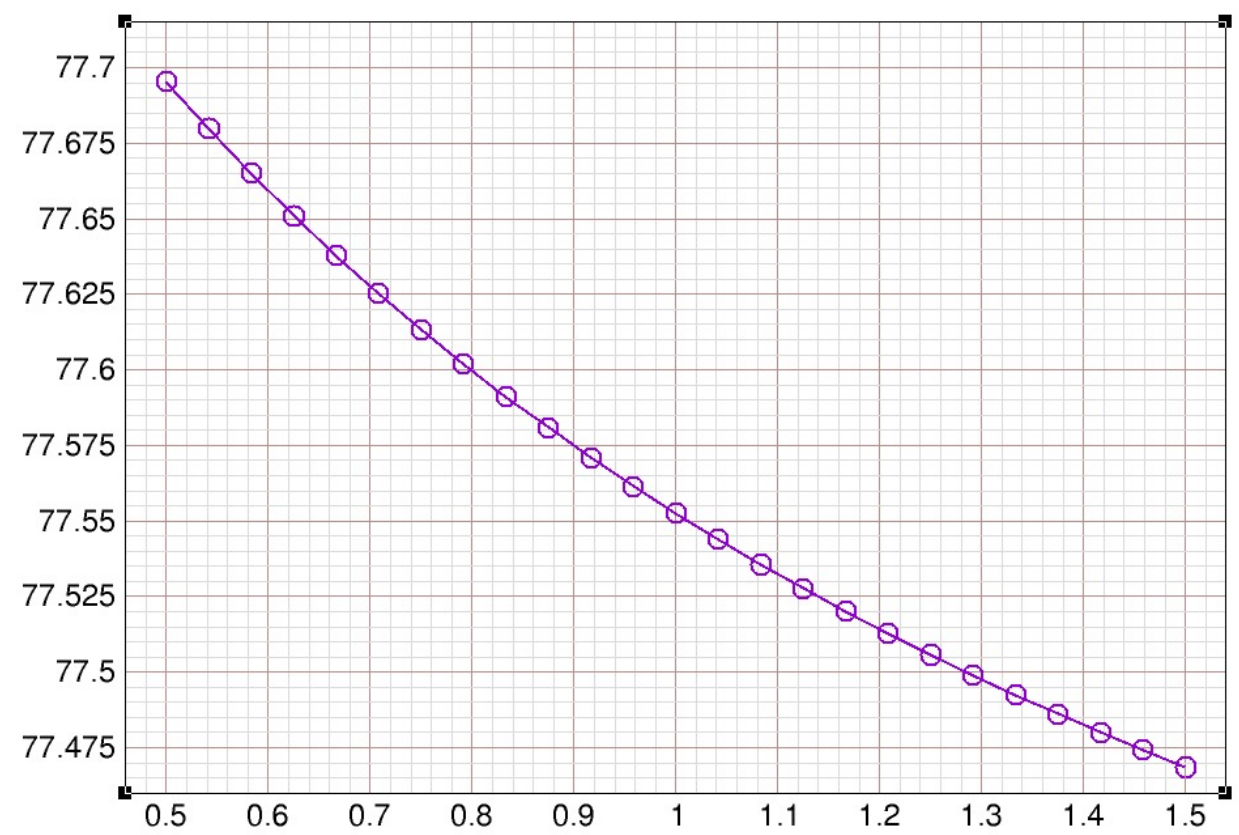

Figure 9: Here the triple-harmonic bucket unstable fixed point phase $\phi_{u}$ (vertical axis) for which bucket area ratio $\mathcal{A}_{3} / \mathcal{A}_{1}$ reaches a minimum has been obtained for several values of the amplitude ratio $A_{1} / A_{2}$ (horizontal axis). The phase is given by equations (127) and (131). These data are indicated by the violet circles. The violet curve connects the circles. 


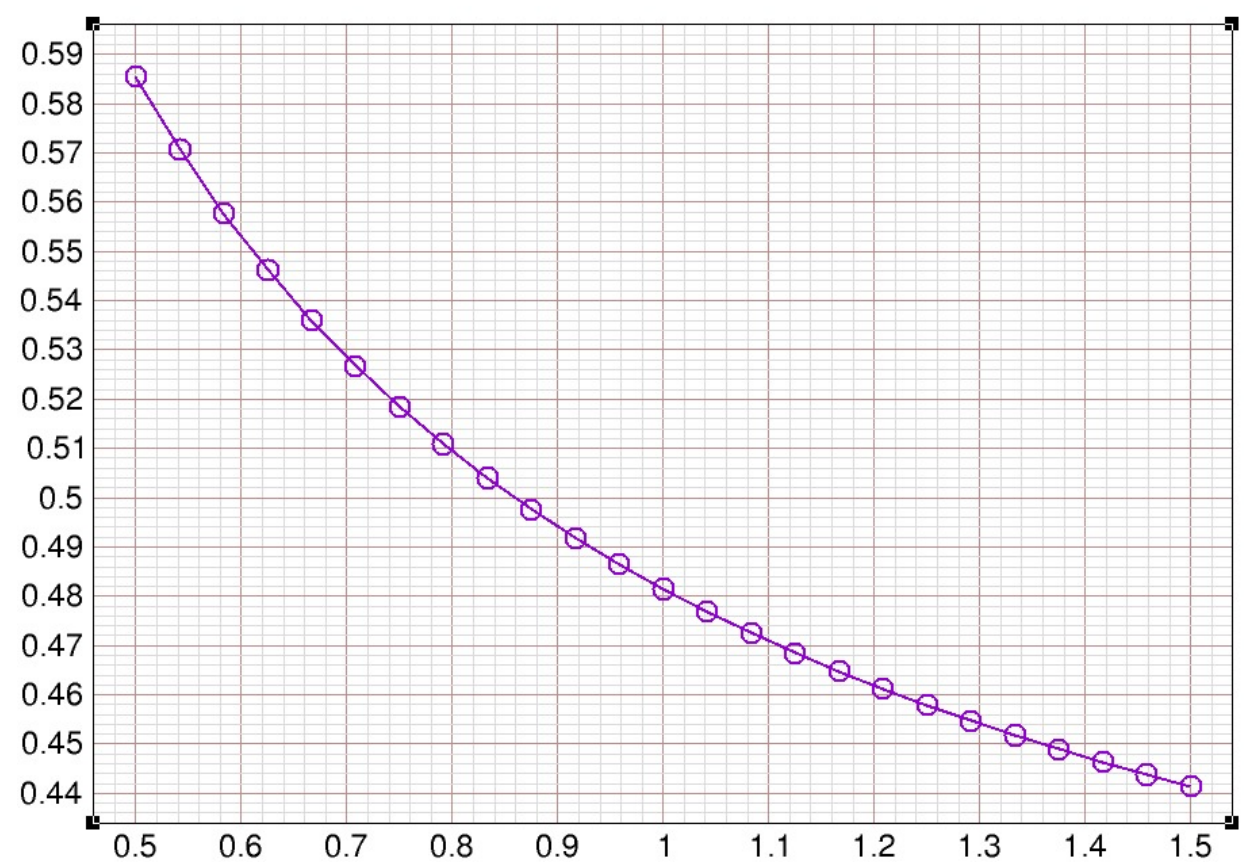

Figure 10: Minimum bucket area ratio $\mathcal{A}_{3} / \mathcal{A}_{1}$ versus amplitude ratio $A_{1} / A_{2}$. Here, again, the calculated data are indicated by the violet circles. The violet curve connects the circles. 


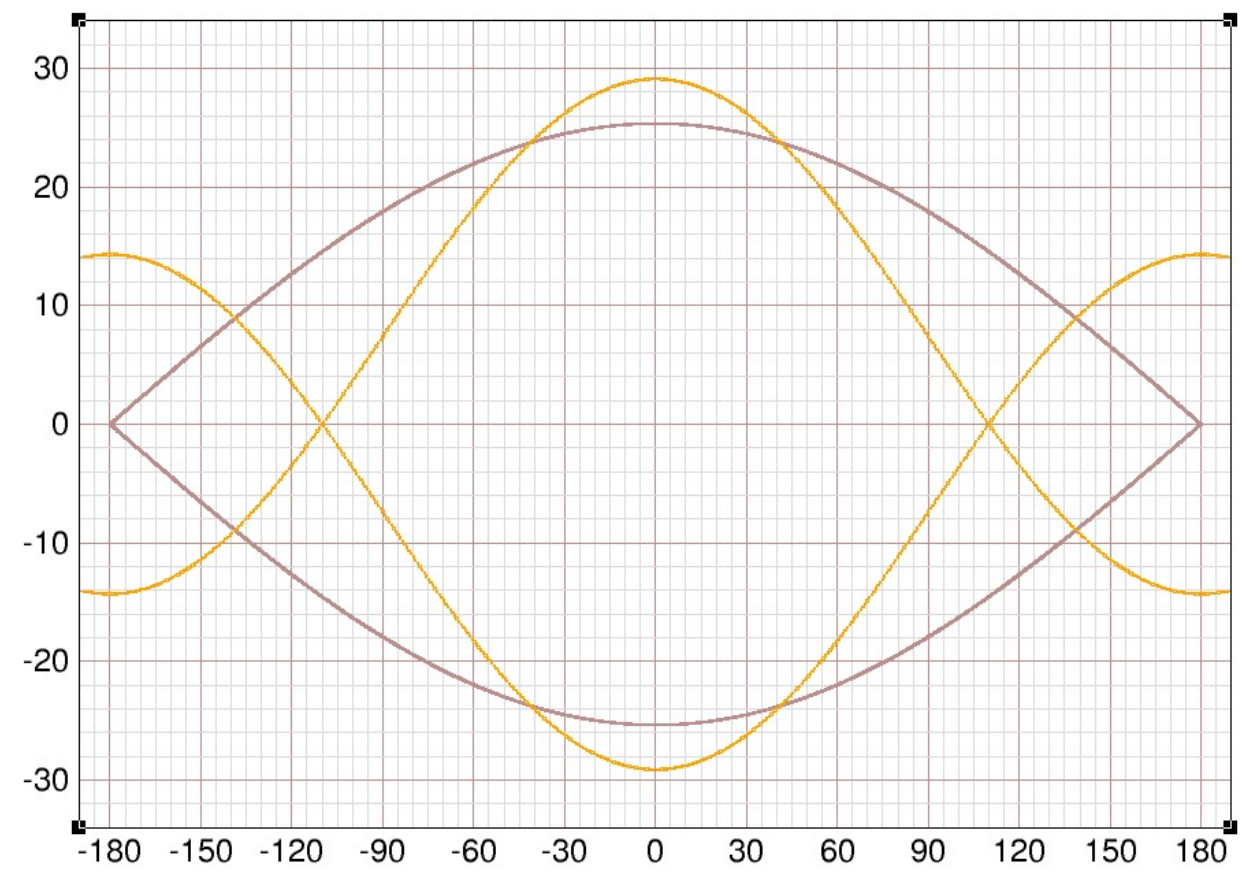

Figure 11: Single and double-harmonic buckets (brown and orange curves) for the case in which the amplitude ratio $A_{1} / A_{2}$ is fixed at $15 / 22$. Using (72) and (91) this gives bucket area ratio $\mathcal{A}_{2} / \mathcal{A}_{1}=0.6826$. 


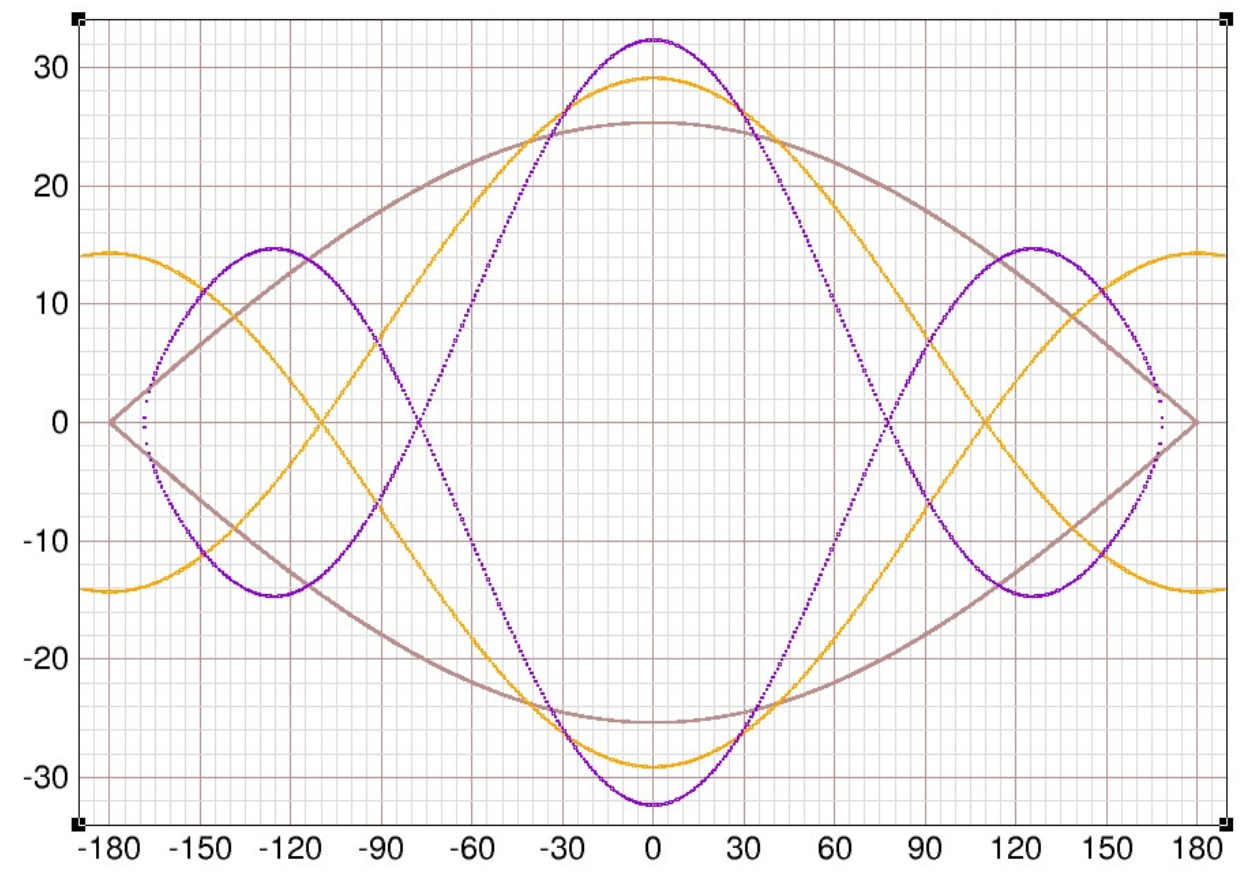

Figure 12: As in the previous figure, the brown and orange curves are the single and double-harmonic buckets for the case in which the amplitude ratio $A_{1} / A_{2}$ is fixed at $15 / 22$. As the harmonic $3 h$ amplitude, $A_{3}$, is raised from zero, the double-harmonic bucket becomes a triple-harmonic bucket. The area of this bucket will decrease from $\mathcal{A}_{3}=\mathcal{A}_{2}$ to a minimum and then begin to increase. The value of $R$ which gives the minimum area can be read off from Figure 8. There one finds $R=1.994$. The corresponding unstable fixed point phase $\phi_{u}$ obtained from Figure $\mathbf{9}$ is 77.633 degrees. The corresponding bucket area ratio $\mathcal{A}_{3} / \mathcal{A}_{1}$ obtained from Figure $\mathbf{1 0}$ is 0.532 . The violet curve is the minimum-area triple-harmonic bucket. 


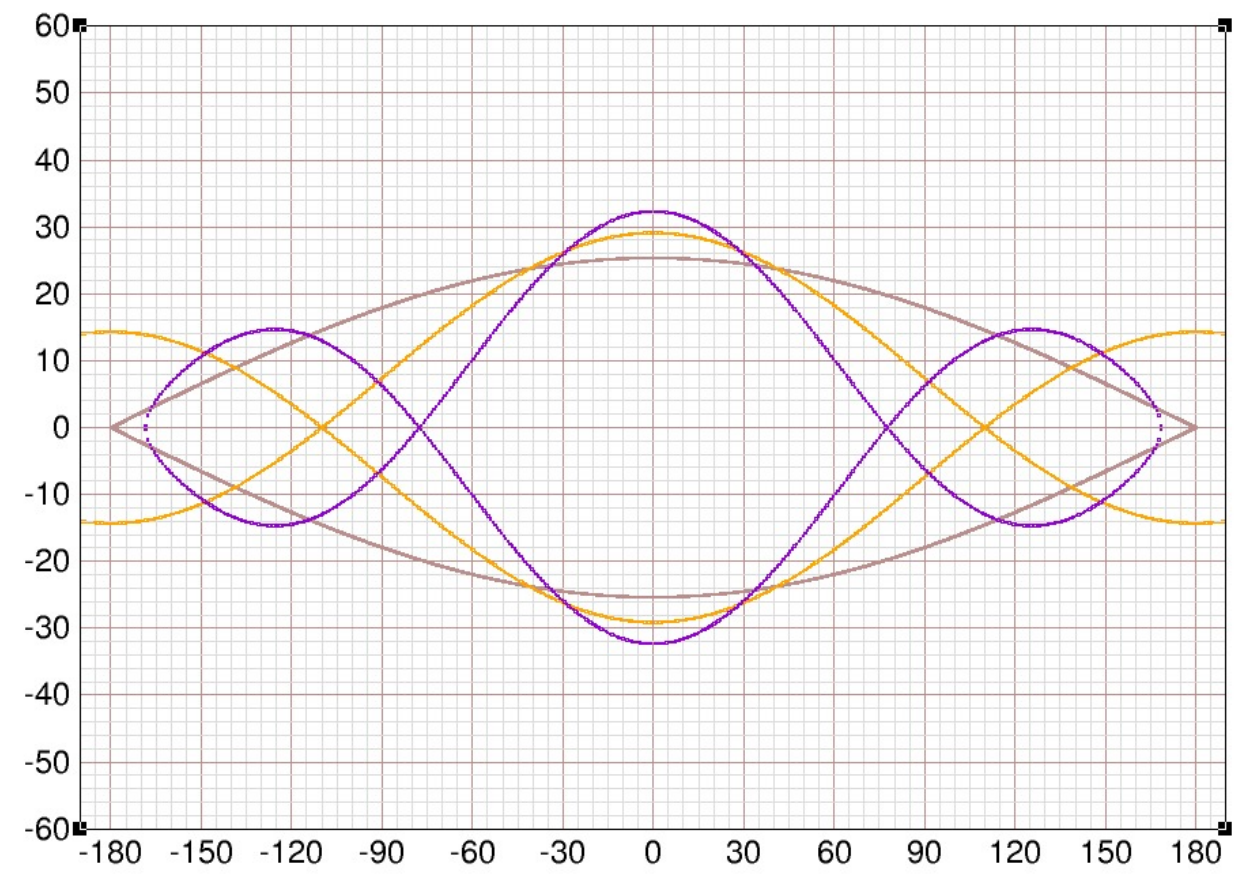

Figure 13: Same as previous figure, but with larger vertical scale. 


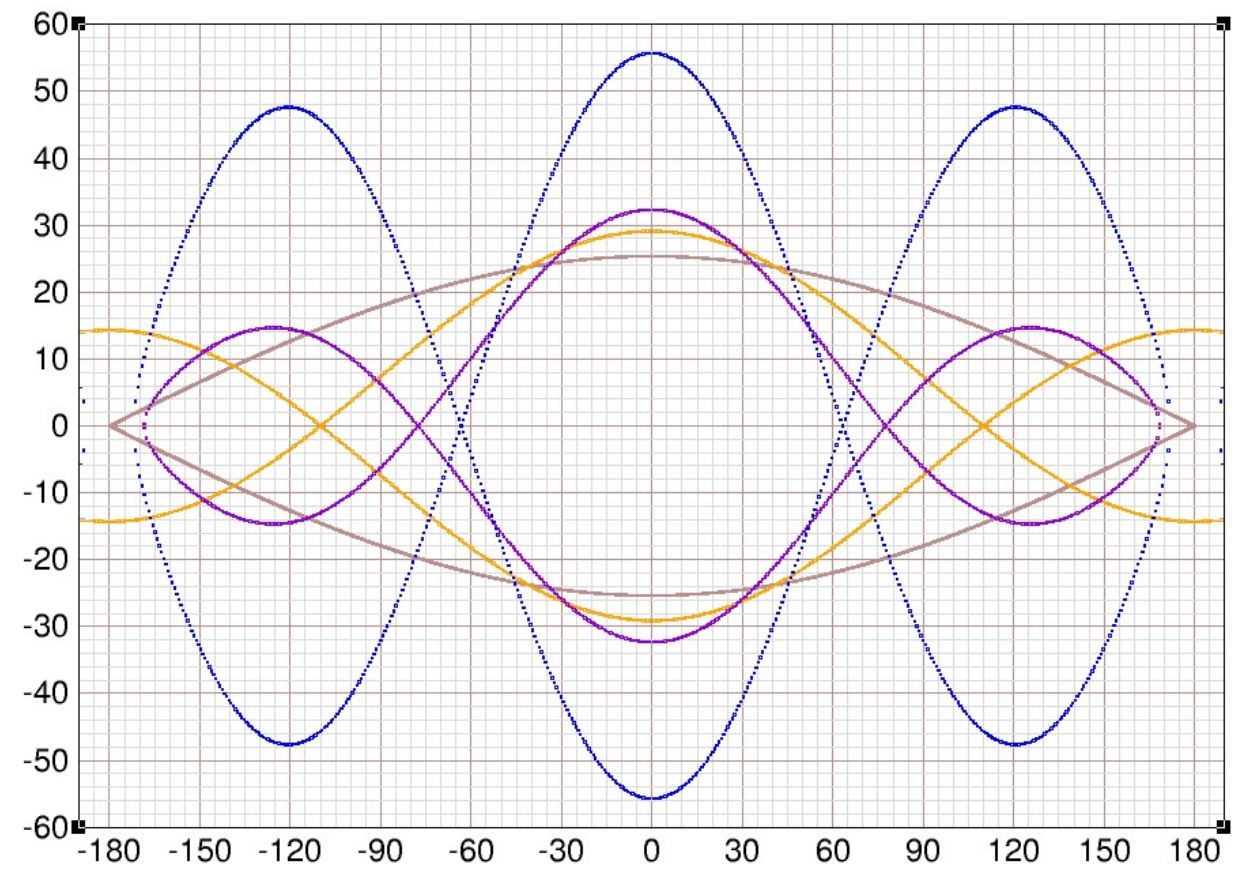

Figure 14: Here, as in the previous two figures, the brown and orange curves are the single and double-harmonic buckets for amplitude ratio $A_{1} / A_{2}=$ $15 / 22$. The violet curve is the corresponding minimum-area triple-harmonic bucket. The amplitude ratio $R=A_{3} / A_{1}$ for this bucket is 1.994 . The blue curve is the triple-harmonic bucket for amplitude ratio $R=180 / 15$. 


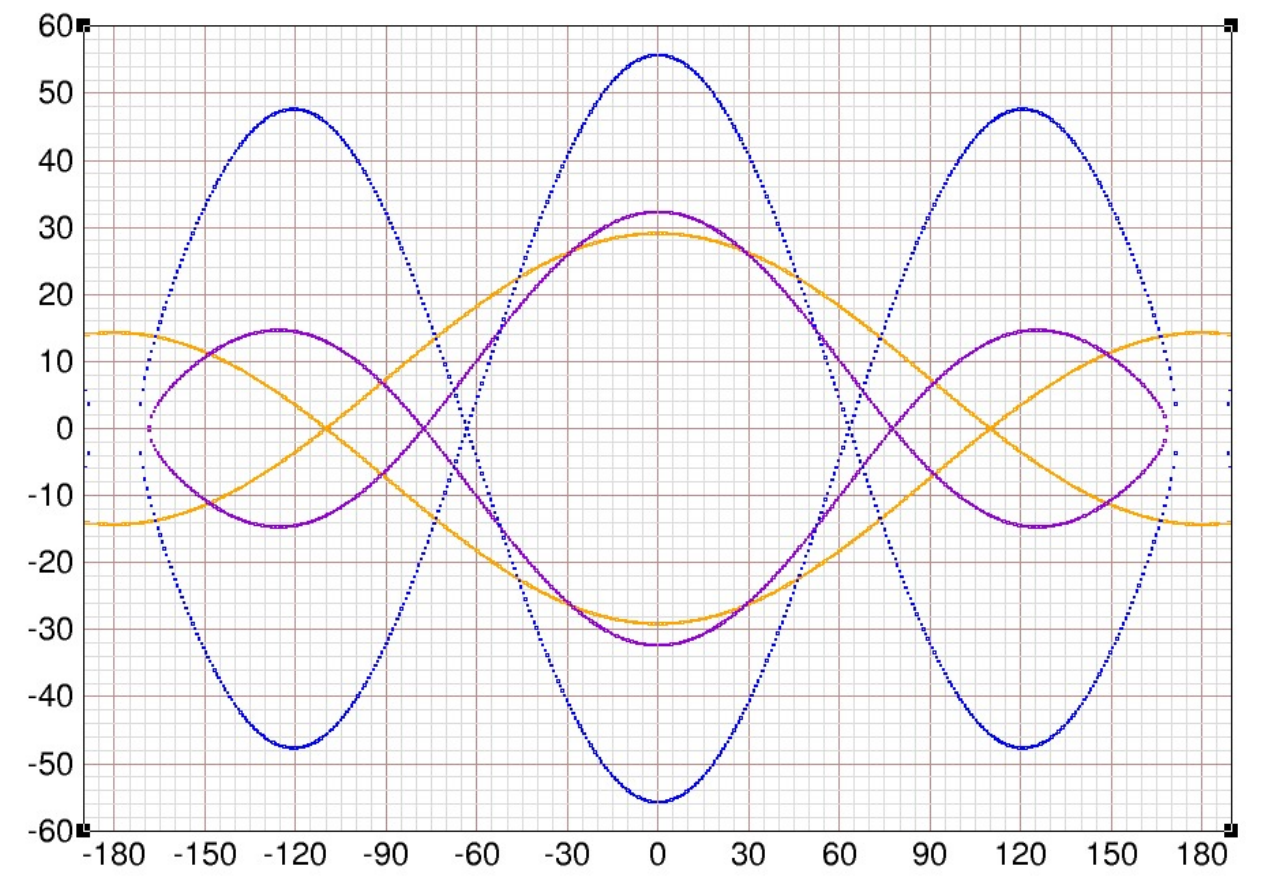

Figure 15: Same as previous figure, but without the single-harmonic bucket. The amplitude ratio $A_{1} / A_{2}$ is fixed at $15 / 22$. The orange, violet, and blue curves are triple-harmonic buckets with $R=0,1.994$, and 180/15, respectively. 


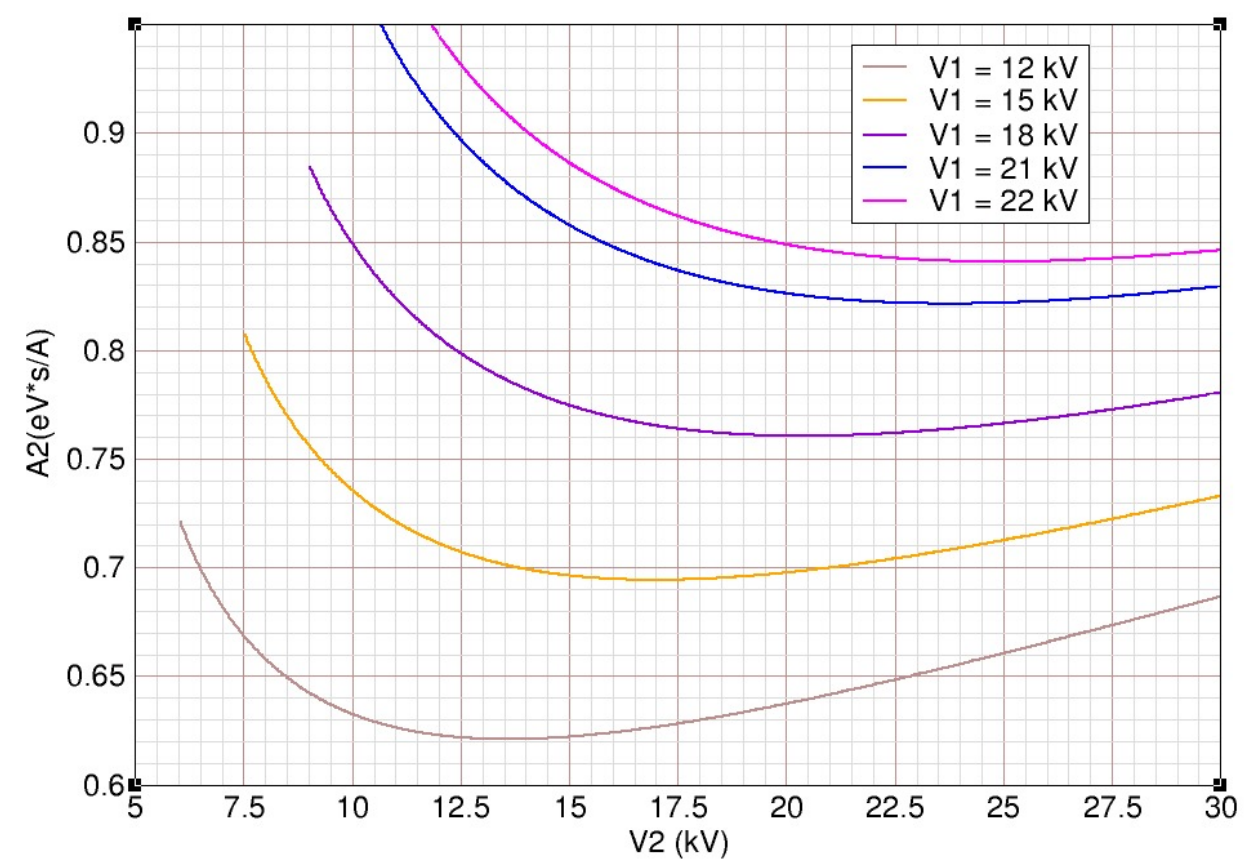

Figure 16: Double-harmonic bucket area $\mathcal{A}_{2}$ obtained for various values of $V_{1}$ and $V_{2}$. The brown, orange, violet, blue, and magenta curves give $\mathcal{A}_{2}$ as a function of $V_{2}$ with $V_{1}$ fixed at $12,15,18,21$, and $22 \mathrm{kV}$, respectively. These curves reach minimum double-harmonic bucket areas $\mathcal{A}_{2}=0.621,0.694$, $0.761,0.822$, and $0.841 \mathrm{eVs}$ per nucleon, respectively. The corresponding voltages $V_{2}$ are $13.6,17.0,20.4,23.7$, and $24.9 \mathrm{kV}$. If, for a given $V_{1}$, the rising second harmonic voltage $V_{2}$ goes through the value for which a minimum is reached, the longitudinal emittance of the merged Au77+ bunch must be less than that minimum if the bunch is to be held in the double-harmonic bucket. This minimum is the acceptance of the double-harmonic portion of the squeeze. If, for example, $V_{1}$ is fixed at $15 \mathrm{kV}$ and the rising voltage $V_{2}$ goes through $17.0 \mathrm{kV}$, then the longitudinal emittance of the merged bunch must be less than $0.694 \mathrm{eV}$ s per nucleon. 


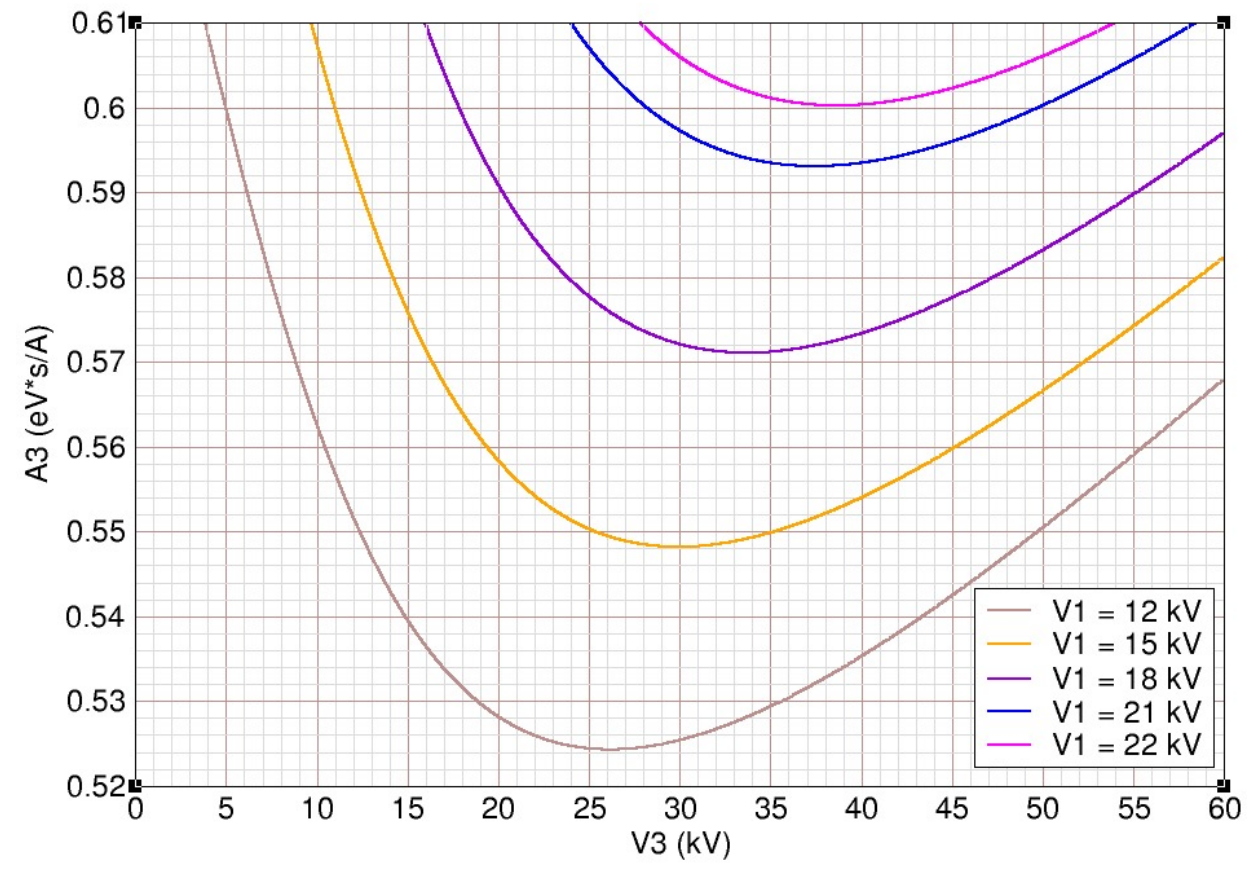

Figure 17: Triple-harmonic bucket area $\mathcal{A}_{3}$ obtained for various values of $V_{1}$ and $V_{3}$ with $V_{2}$ fixed at $22 \mathrm{kV}$. The brown, orange, violet, blue, and magenta curves give $\mathcal{A}_{3}$ as a function of $V_{3}$ with $V_{1}$ fixed at $12,15,18$, 21 , and $22 \mathrm{kV}$, respectively. These curves reach minimum triple-harmonic bucket areas $\mathcal{A}_{3}=0.524,0.548,0.571,0.593$, and $0.600 \mathrm{eV}$ s per nucleon, respectively. The corresponding voltages $V_{3}$ are 26.2, 29.9, 33.7, 37.4, and $38.7 \mathrm{kV}$. If, for a given $V_{1}$, the rising third harmonic voltage $V_{3}$ goes through the value for which a minimum is reached, the longitudinal emittance of the merged Au77+ bunch must be less than that minimum if the bunch is to be held in the triple-harmonic bucket. This minimum is the acceptance of the triple-harmonic portion of the squeeze. If, for example, $V_{1}$ is fixed at 15 $\mathrm{kV}$ and the rising voltage $V_{3}$ goes through $29.9 \mathrm{kV}$, then the longitudinal emittance of the merged bunch must be less than $0.548 \mathrm{eV}$ s per nucleon.

The minimum bucket areas $0.524,0.548,0.571 \mathrm{eVs}$ per nucleon obtained here for $V_{1}=12,15,18 \mathrm{kV}$, respectively, are in good agreement with the corresponding longitudinal acceptances $0.526,0.550,0.574$ obtained by simulation in [2]. 


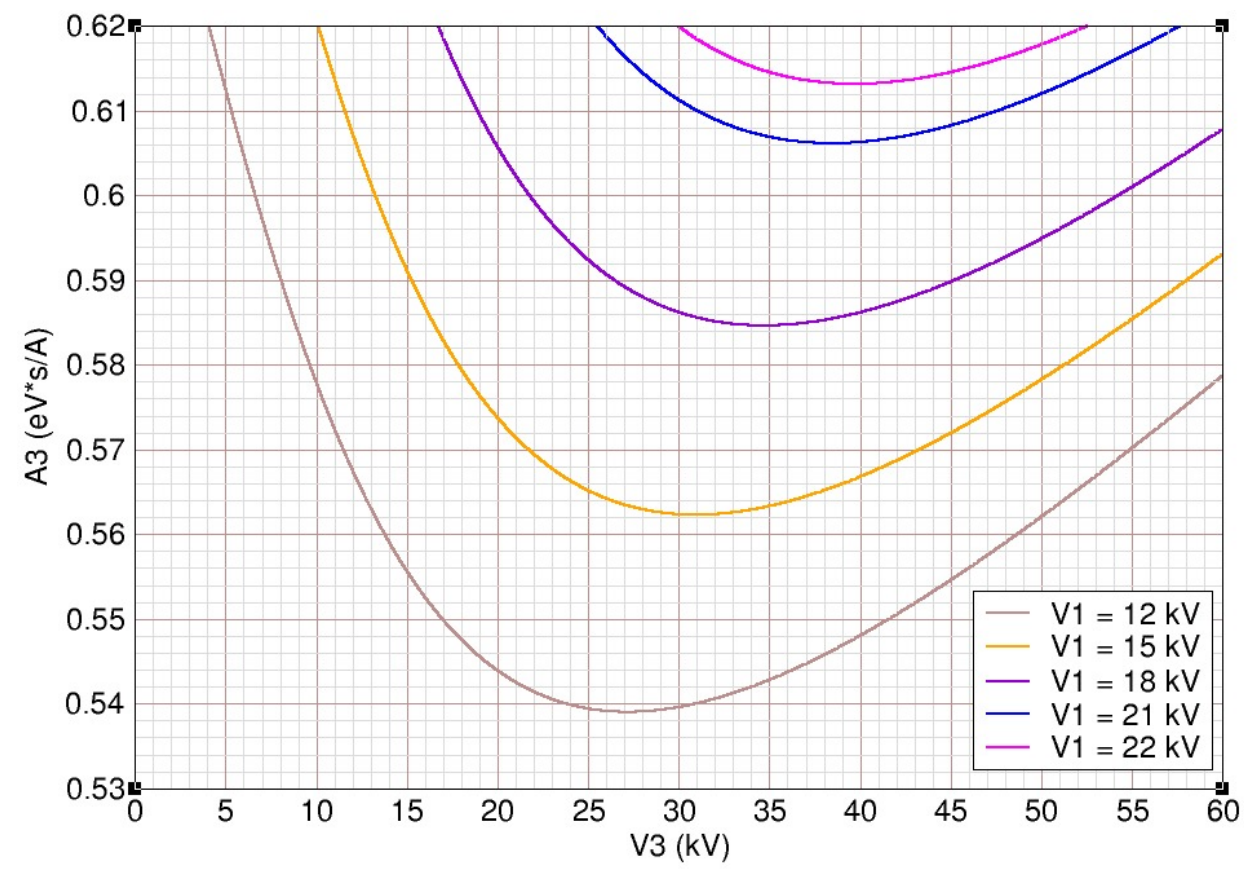

Figure 18: Triple-harmonic bucket area $\mathcal{A}_{3}$ obtained for various values of $V_{1}$ and $V_{3}$ with $V_{2}$ fixed at $24 \mathrm{kV}$. The brown, orange, violet, blue, and magenta curves give $\mathcal{A}_{3}$ as a function of $V_{3}$ with $V_{1}$ fixed at 12, 15, 18, 21, and $22 \mathrm{kV}$, respectively. These curves pass through minimum triple-harmonic bucket areas $\mathcal{A}_{3}=0.539,0.562,0.585,0.606$, and $0.613 \mathrm{eVs}$ per nucleon, respectively. The corresponding voltages $V_{3}$ are $27.2,30.9,34.7,38.4$, and $39.7 \mathrm{kV}$. As before, if, for a given $V_{1}$, the rising voltage $V_{3}$ goes through the value for which a minimum is reached, the longitudinal emittance of the merged Au77+ bunch must be less than that minimum if the bunch is to be held in the triple-harmonic bucket. In this case, if $V_{1}$ is fixed at $15 \mathrm{kV}$ then the longitudinal emittance of the merged bunch must be less than $0.562 \mathrm{eV} \mathrm{s}$ per nucleon. 


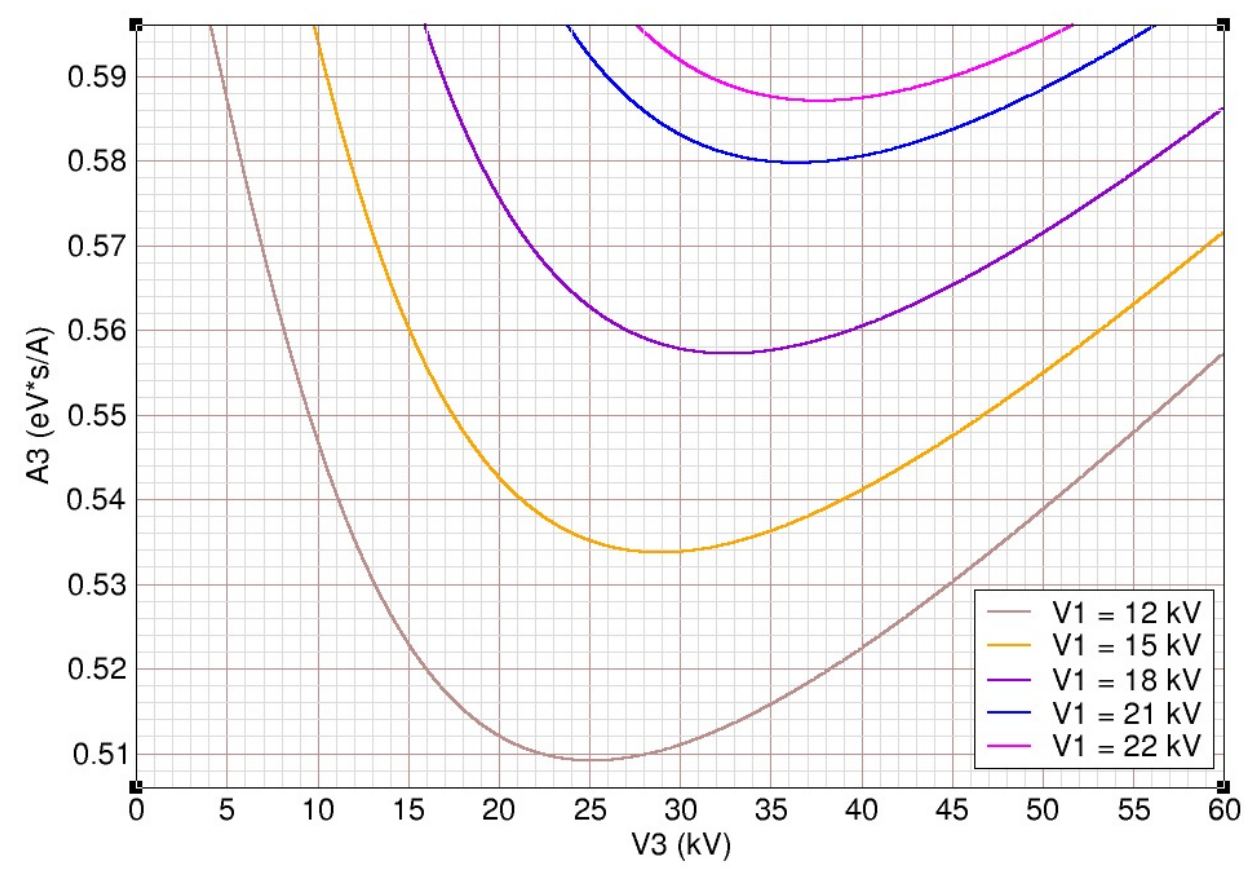

Figure 19: Triple-harmonic bucket area $\mathcal{A}_{3}$ obtained for various values of $V_{1}$ and $V_{3}$ with $V_{2}$ fixed at $20 \mathrm{kV}$. The brown, orange, violet, blue, and magenta curves give $\mathcal{A}_{3}$ as a function of $V_{3}$ with $V_{1}$ fixed at $12,15,18,21$, and $22 \mathrm{kV}$, respectively. These curves pass through minimum triple-harmonic bucket areas $\mathcal{A}_{3}=0.509,0.534,0.557,0.580$, and $0.587 \mathrm{eV} \mathrm{s}$ per nucleon, respectively. The corresponding voltages $V_{3}$ are 25.1, 28.9, 32.7, 36.4, and $37.7 \mathrm{kV}$. As before, if, for a given $V_{1}$, the rising voltage $V_{3}$ goes through the value for which a minimum is reached, the longitudinal emittance of the merged Au77+ bunch must be less than that minimum if the bunch is to be held in the triple-harmonic bucket. In this case, if $V_{1}$ is fixed at $15 \mathrm{kV}$ then the longitudinal emittance of the merged bunch must be less than $0.534 \mathrm{eV} \mathrm{s}$ per nucleon. 